ISSN: 0514-7336

DOI: http://dx.doi.org/10.14201/zephyrus2014731343

\title{
LAS INDUSTRIAS ARCAICAS DEL ROSELLÓN (SUR DE FRANCIA): CORRELACIÓN GEOCRONOLÓGICA CON LAS TERRAZAS DE LOS RÍOS TÊT, TECH Y AGLY
}

\section{Archaic Industries of Roussillon (South of France): Geochronological Correlation with the Terraces of the Têt, Tech and Agly Rivers}

\author{
Joan GARCIA GARRIGA
}

Institut Català de Paleoecologia Humana i Evolució Social. C/ Marcel.li Domingo, s/n. Campus Sescelades URV. Edifici W3. 43007 Tarragona. Correo-e: jgarc338@xtec.cat

Recepción: 03/04/2012; Revisión: 23/07/2012; Aceptación: 13/02/2014

BIBLID [0514-7336 (2014) LXXIII, enero-junio; 17-43]

Resumen: Este trabajo analiza la industria lítica del Paleolítico inferior de las terrazas del Rosellón, en el SE de Francia. La revisión geomorfológica realizada de los niveles fluviales permite correlacionar estas industrias con la geocronología de las terrazas, formadas entre el Pleistoceno inferior final y medio, estableciendo un marco cronocultural regional. Mediante el análisis tecnológico de las industrias derivado del concepto de chaîne opératoire y el estudio de las pátinas, se diferencian los Modos técnicos y se discriminan los aspectos tecnológicos de las industrias según los procesos de configuración y explotación. En la secuencia fluvial se ha identificado el Modo 1 en la terraza de Mas Ferréol y el Modo 2 en las de la Plane d'en Bourgat y Butte du Four-Llabanère, observándose una continuidad tecnológica, con perduraciones de cantos tallados - choppers y chopping-tools- y cadenas de explotación poco organizadas - poliedros-. Este sustrato tecnológico es común al de los yacimientos coetáneos del NE de la Península Ibérica y SE de Francia, aunque se ha detectado la existencia de un margen de variabilidad entre las industrias de las distintas regiones.

Palabras clave: Paleolítico inferior. Chaîne opératoire. Continuidad tecnológica. Estasis tecnológica. Modo 1. Modo 2.

ABSTRACT: This article studies the lithic industry of the Early Palaeolithic from the terraces of the Roussillon, in the S of France. The geomorphological revision of the flood levels allows these industries to be related to the geochronology of the terraces which were formed in between the late Early Pleistocene and the Middle Pleistocene, and thus allows a regional chrono-cultural framework to be established. By analyzing the technology of the lithic industry derived from the concept of chaine opératoire and studying the patinas, the technical Modes and the technological aspects of the processes related to configuration and knapping sequences are differentiated. In the fluvial sequence, Mode 1 is documented in the terrace of Mas Ferréol and Mode 2 is identified in the Plane d'en Bourgat and Butte du Four-Llabanère, thus showing a technological continuity in the form of choppers and chopping-tools and poorly organized chains of exploitation (polyhedral). This technological substratum is common to the coeval sites from the NE of the Iberian Peninsula and the S of France, although with a margin of variability between the industries from the different regions.

Key words: Early Palaeolithic. Chaîne opératoire. Technological continuity. Technological stasis. Mode 1. Mode 2. 


\section{Introducción}

En este artículo hemos analizado la industria lítica recuperada en prospecciones sistemáticas en las terrazas fluviales de la llanura del Rosselló -S de Francia- ${ }^{1}$. Los sucesivos cambios climáticos acontecidos durante el Pleistoceno provocaron una progresiva acción erosiva y de deposición de sedimentos que formaron seis terrazas al paso del Agly y del Têt, las tres más antiguas con industrias del Paleolítico inferior. El registro lítico hallado en estos niveles fluviales permite obtener una visión diacrónica sobre la tecnología del Paleolítico inferior en la región, comprendiendo un abanico temporal que abarca desde finales del Pleistoceno inferior hasta el Pleistoceno medio. Se trata de 93 enclaves en superficie, la mayoría localizaciones aisladas y dispersas con escasos efectivos recuperados durante prospecciones sistemáticas, aunque algunas otras como Rodes -607 objetoso Mas Ferréol -119- son más importantes (Figs. 1, 3 y 4). Hasta ahora, debido a problemas de interpretación relacionados con la compleja geomorfología del sistema de terrazas, estas industrias en superficie se habían relacionado con niveles fluviales equivocados, llevando a interpretaciones cronológicas erróneas de las industrias (CollinaGirard, 1976a, 1976b, 1976c, 1978; de Lumley, 1969a, 1969b, 1971, 1976; de Lumley et al., $1976)^{2}$.

Una vez subsanados estos problemas, presentamos una revisión geomorfológica del sistema de terrazas con el objetivo de clarificar el contexto de los niveles fluviales en el que se encontraron las industrias. Asimismo, hemos llevado a cabo una correlación geocronológica de las terrazas con sus industrias, para poder disponer de un marco cronocultural de referencia más preciso para las ocupaciones humanas de la región. Los criterios fundamentales para establecerlo proceden de la

\footnotetext{
${ }^{1}$ Agradezco la colaboración en este estudio del Centre Européen de Recherches Préhistoriques de Tautavel (CERP), especialmente de su presidente, el profesor $\mathrm{H}$. de Lumley, y de D. Dainat por la cesión de material fotográfico.

2 Cf. también Collina-Girard, J.: Les industries archaïques sur galet des terrasses quaternaires de la plaine $d u$ Roussillon (Pyrénées-Orientales, France). Tesis doctoral inédita, presentada en 1975 en la Univ. de Provence.
}

revisión que hemos llevado a cabo a partir de los estudios altimétricos de las plataformas fluviales, en relación con el nivel actual del río y de su composición litológica (Bourcart, 1945; Got, 1967; Barrière, 1969; Barrière y Michaux, 1973; Clauzon, 1984; Giret, 1995; Duvail et al., 2001)33. A partir de la información actual, los enclaves con industria del Paleolítico inferior quedan adscritos a tres terrazas principales: la de Mas Ferréol, de finales del Pleistoceno inferior; la de la Plane d'en Bourgat, datada entre finales del Pleistoceno inferior e inicios del medio, y la de la Butte du FourLlabanère, del Pleistoceno medio. Esta amplia diacronía nos ha permitido analizar los cambios tecnológicos sucedidos durante el Paleolítico inferior regional, donde en la más antigua de estas terrazas se documenta una industria de Modo $1 \mathrm{y}$ en las dos más recientes de Modo 2.

Esta información resulta relevante para contextualizar las ocupaciones humanas coetáneas establecidas en las regiones próximas, como las del vecino yacimiento de la Caune de l'Arago, en Tautavel (Barsky y de Lumley, 2010), las de los cercanos asentamientos, también sobre sistemas de terrazas, de los ríos Garona (Bruxelles et al., 2003), Dordoña y Lot (Turq, 2000) ${ }^{4}$, en el s de Francia, o las del río Ter y depresión de la Selva, en Gerona (García Garriga, 2008, 2010, 2011). La comparación tecnológica de las industrias del Paleolítico inferior de estas regiones también nos puede permitir establecer un modelo sobre el radio de acción de los grupos humanos en la captación de las materias primas e interpretar la variabilidad tecnológica existente entre los yacimientos de las diversas áreas.

${ }^{3}$ Cf. también Calvet, M.: Morphogenèse d'une montagne méditerranéenne. Les Pyrénées Orientales. Tesis doctoral inédita, presentada en 1994 en el Muséum National d'Histoire Naturelle de París; Debals, B. (1998): Étude sedimentologique des formations quaternaires des bassins des fleuves cotiers du Roussillon (France): Valles de la Têt et $d u$ Tech. Tesis doctoral inédita, presentada en 1998 en la Univ. de Perpignan, y Monaco, A.: Contribution à l'étude géologique et sédimentologique du plateau continental $d u$ Roussillon (Golfe du Lion). Tesis doctoral inédita, presentada en 1971 en la Univ. de Montpellier.

${ }^{4}$ Cf. también Turq, A.: Le Paléolithique inferieur et moyen entre les valles de la Dordogne et du Lot. Tesis doctoral inédita, presentada en 1992 la Univ. de Bordeaux I. 


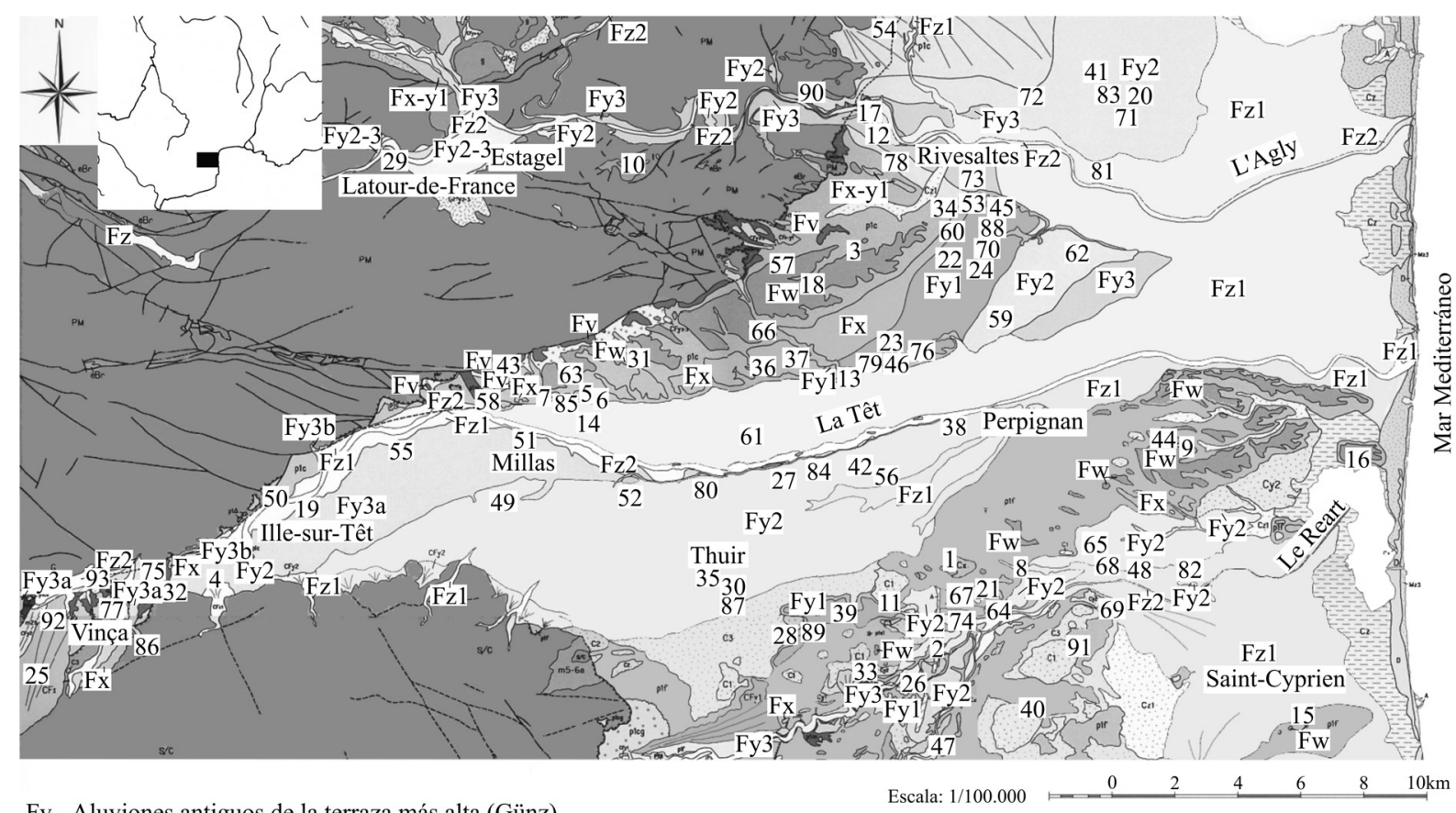

Fv - Aluviones antiguos de la terraza más alta (Günz)

$\mathrm{Fw}$ - Aluviones antiguos de la terraza alta (Mindel)

Fx - Aluviones antiguos de las terrazas del Riss

Fyl- Aluviones antiguos de las terrazas del Würm antiguo

Fx-y1 - Aluviones antiguos de las terrazas del Würm antiguo-Riss

Fy2 - Aluviones antiguos de las terrazas del Würm reciente

$1 / 100.000$

Fy $3 b$ - Aluviones antiguos de las terrazas tardiglaciares (nivel inferior) Fy3 - Aluviones antiguos de las terrazas tardiglaciales

Fy2-3 - Aluviones antiguos de las terrazas del Würm reciente-Tardiglaciar Fz - Aluviones holocenos

Fz1 - Aluviones recientes de las terrazas bajas holocenas

Fy3a - Aluviones antiguos de las terrazas tardiglaciares (nivel superior) Fz2 - Aluviones actuales

FIG. 1. Situación de los enclaves del Paleolitico inferior del Rosellón en relación a las terrazas del Têt y Agly -la localización correspondiente a la terraza de Mas Ferréol es la 43; las de la terraza de la Plane d'en Bourgat son la 1, 3, 9, 15, 16, 18, 31, 44, 47, 57, 63 y 66, y el resto pertenecen a la terraza de la Butte du Four-Llabanère-: 1) Aire des Pavillons; 2) Baixades; 3) Baixas; 4) Bouleternére; 5) Butte 124; 6) Butte 69; 7) Butte du Four; 8) Cabane Auriol; 9) Cabestany; 10) Calce Cabanac; 11) Canohés; 12) Cimetière d'Espira; 13) Coll de la Guille; 14) Corneilla-la-Riviére; 15) Saint-Cyprien; 16) Esparrou-Canet; 17) Espira de l'Agly; 18) Gourg; 19) Ille-surTêt; 20) Jaç Moscatars; 21) La Devéze Ouest; 22) La Femme Morte; 23) La Garriga; 24) La Llabanère; 25) La Prunette; 26) La Resclose; 27) Le Soler; 28) La Torra; 29) Latour de France; 30) Le Boulou; 31) Le Correch; 32) Les Anecs; 33) Les Baguères; 34) Les Sedes; 35) Llupia Mas Saint Sauveur; 36) Manadeil; 37) Manadeil Relais PTT; 38) Mas Bruno; 39) Mas Canterrene; 40) Mas de la Prada; 41) Mas del Crest; 42) Mas Ducup; 43) Mas Ferréol; 44) Mas Ferrer; 45) Mas Rombau; 46) Mas Romeu; 47) Mas Sabole; 48) Mas Sante Thérèse; 49) Mas Selve; 50) Mata Roudoume; 51) Millas; 52) Moulin de Saint Feliu; 53) Moulin du Souffre; 54) Moutou-la-Joliette; 55) Néfiach; 56) Orla; 57) Oums; 58) Pascal; 59) Route de Pia; 60) Peyrestortes; 61) Pézilla de la Rivière; 62) Pia Route de Bompas; 63) Plane d'en Bourgat; 64) Pollestres; 65) Porte d'Espagne; 66) Puech de la Boule; 67) Réservoir; 68) Rive Droite du Reart; 69) Rive Gauche du Reart; 70) Rivesaltes le Jaç; 71) Rivesaltes Moscatars; 72) Rivesaltes Paniçó; 73) Rivesaltes Saint Bernard; 74) Route National 117 Réservoir; 75) Rodés; 76) Route de Saint Estéve; 77) Route de Vinça; 78) Route National d'Espira; 79) Saint Estève; 80) Saint Féliu Briqueterie; 81) Saint Pierre; 82) Saleilles; 83) Salses Mas Burguére; 84) Sainte Eugénie; 85) Ter de la Têt la Baillobère; 86) Ternére; 87) Terrasse du Boulou; 88) Torrella; 89) Trouillas; 90) Vespeille Mas Crémat; 91) Villeneuve-la-Raho; 92) Vinça y 93) Vinça Saint Pierre (cf. la columna de la Tabla 3 de Clauzon, 1984 y Duvail et al., 2001) 


\section{Las terrazas de los ríos Têt, Tech y Agly}

\subsection{Estratigrafia del sistema de terrazas}

La cuenca de la llanura del Rosellón forma parte de los Pirineos Orientales, extendiéndose entre el macizo de las Corbières al N, los Pirineos al o y al s y el mar Mediterráneo al E. El territorio que la forma está drenado por pequeños ríos de escaso caudal: el Agly, el más septentrional, que recibe sus aguas de las Corbières; el Têt, que nace en el macizo del Carlite e irriga el valle del Conflent, y el Tech, en la parte más meridional, que discurre por el valle del Vallespir. Todos presentan direcciones paralelas y se disponen sobre el terreno con una orientación O-E. Las seis terrazas fluviales

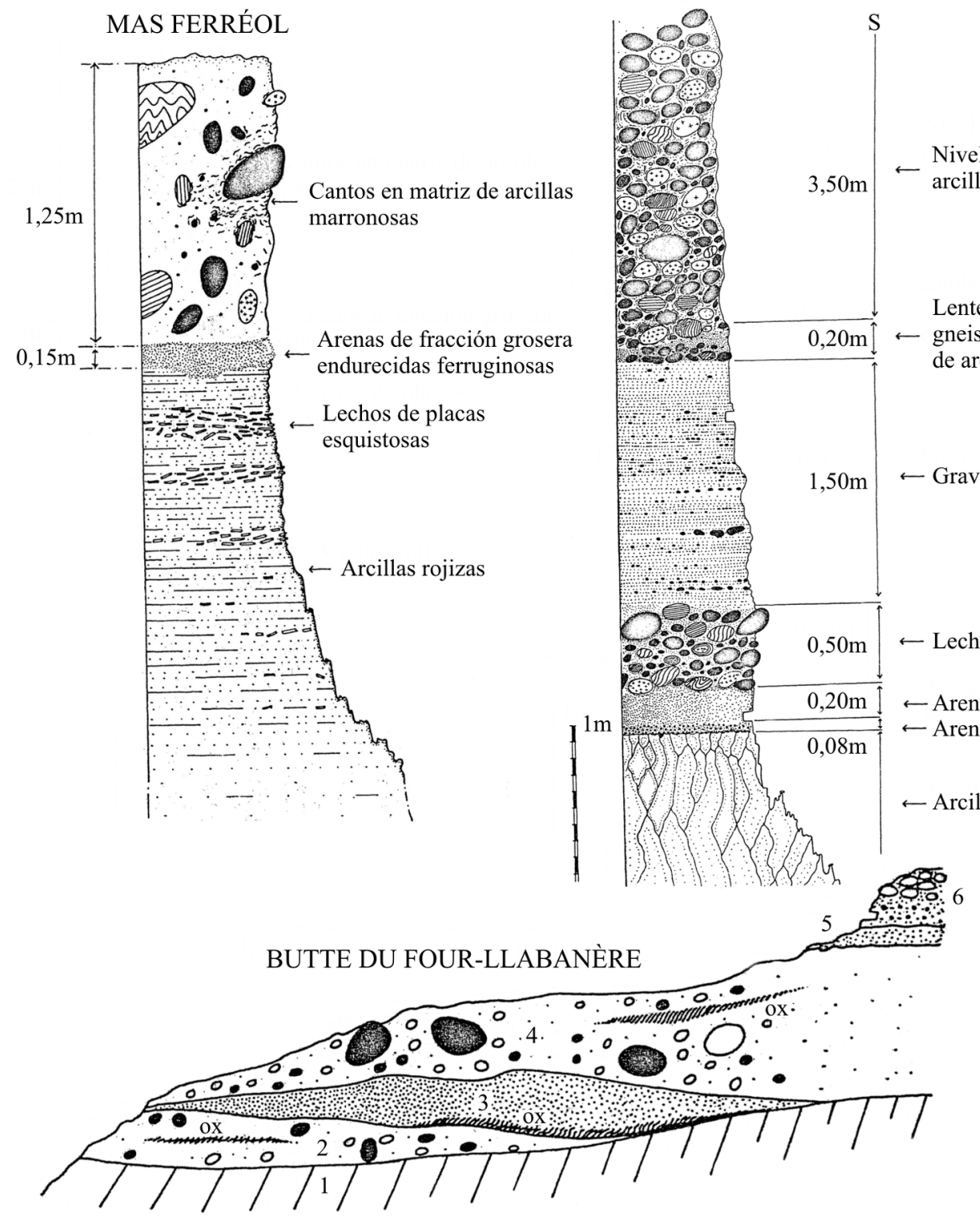

1 Plioceno

2 Primer lentejón de cantos 3 Arenas rojizas

4 Segundo lentejón de cantos

5 Arenas rojizas

6 Tercer lentejón de cantos ox Lechos de óxidos

FIG. 2. Secciones estratigráficas de Mas Ferréol y de Ravin des Oums, en la Plane d'en Bourgat, y de la Butte du FourLlabanère (a partir de Collina-Girard, op. cit. n. 2: 100, 109 y 134). 
que se formaron en la cuenca neógena de la llanura del Rosellón por la acción erosivo-sedimentaria de estos ríos, especialmente del Têt, se sitúan en un área delimitada entre las localidades de Prades y Perpinán. La totalidad de yacimientos y localizaciones paleolíticas están diseminadas sobre la superficie de estas terrazas, mayoritariamente sobre la ribera izquierda del Têt. El hecho de que se trate de industria hallada en superficie en prospecciones impide su adscripción a niveles estratigráficos concretos, por lo que debe correlacionarse genéricamente con los paquetes sedimentarios de sus respectivas terrazas (Fig. 1).

La terraza de Mas Ferréol, en la que existe un único enclave arqueológico, se conserva al $\mathrm{N}$ de la localidad de Millas, sobre la ribera izquierda del Têt. Este depósito sedimentario, que está actualmente muy desmantelado, marca el inicio del Pleistoceno en la cuenca del Têt. Sólo se ha conservado preservada de la erosión una parte del depósito, con una potencia de $4 / 5 \mathrm{~m}$, que se sobrepone al sustrato esquistoso y a los niveles de coluviones pliocenos (Fig. 2). Es importante la presencia de bloques y cantos de cuarzo procedentes del desmantelamiento del macizo granítico de Millas, que suelen tener más de $1 \mathrm{~m}$ de longitud. Igualmente abundan otros materiales aluviales de fracciones grandes, como los cantos de granito y gneis y las plaquetas de esquisto, lo que sugiere que este depósito se formó durante una dinámica torrencial.

Mata Roudoume es una plataforma aluvial que forma parte de la de Mas Ferréol y que se alzó y separó de ésta después del desplazamiento neotectónico que provocó la falla de las Orgues de Ille-sur-Têt ${ }^{5}$. Este afloramiento fluvial, situado en la misma ribera del Têt, está formado por depósitos pliocenos integrados por niveles arenosos blanquecinos rubefactados. Además, están intercalados por niveles arcillosos y acumulaciones dispersas y continuas de cantos. El último de estos niveles de cantos corona las formaciones pliocenas conformando un pequeño promontorio. La parte superior está constituida por bloques redondeados de cuarzo de una talla media superior a $1,5 \mathrm{~m}$ que se asocian a niveles arenosos arcósicos blancos del Plioceno. Su disposición

${ }^{5}$ Calvet: op. cit. n. 3. estratigráfica cruzada y poco homogénea a nivel litológico nos indica que el régimen fluvial sería torrencial, lo que demuestra su pertenencia a la misma estructura fluvial que Mas Ferréol. El contacto del Plioceno y el zócalo es visible sobre el lado izquierdo del Têt, donde el sustrato está constituido por un antiguo escarpado dominado por niveles graníticos en posición derivada infrapuestos a las arenas arcósicas pliocenas. El punto de contacto está marcado por un nivel heterogéneo de grandes bloques graníticos dispuestos en el sedimento.

En la misma ribera se sitúa a continuación la terraza de la Plane d'en Bourgat, que contiene 12 enclaves arqueológicos. Se trata de una formación aluvial bien desarrollada en la región de Cabestany que presenta un importante nivel de desmantelamiento y eolización, especialmente en el borde derecho del río Têt. Litológicamente se caracteriza por la destacada frecuencia de bloques de cuarzo - granitos, gneis y esquistos fueron destruidos por la intensa alteración que afectó esta formación-, a menudo en un avanzado estado de eolización y con pátinas rojizas (Fig. 2). Localmente aparecen interrumpidos por niveles con una matriz arenosa de tonalidades rojizas y de fracción gruesa. Este importante componente grueso marca una continuación del régimen torrencial, iniciado con la deposición de los sedimentos de la terraza de Mas Ferréol. Sin embargo, la dinámica hidrográfica sigue siendo difusa, tal y como demuestra la existencia de un único lecho aluvial.

Las terrazas medias dan cabida a la mayoría de enclaves y localizaciones arqueológicas de la región -un total de $80-$. La Butte du Four-Llabanère está más afectada por la erosión que las anteriores y es la primera que se conserva en el margen derecho del Têt, aunque sólo en algunas franjas dispersas entre Soler y Perpiñán (Fig. 2). Constituye una extensa y continua plataforma aluvial plana, emplazada en su mayor parte sobre la orilla izquierda del Têt. La configuración de estas formaciones estuvo notablemente afectada por los procesos erosivos, en los que el curso fluvial del Têt se individualizó ${ }^{6}$. A su paso

${ }^{6}$ Calvet: op. cit. n. 3. 
por Saint-Estève, la terraza se convierte en una torrentera que erosiona las formaciones pliocenas infrapuestas.

La composición petrográfica del sistema de terrazas del Têt y Agly es relativamente homogénea. De hecho, las rocas que aparecen sobre los terrenos sólo varían por la proporción con la que aparecen en las diversas terrazas ${ }^{7}$. En general, los materiales característicos son los cuarzos, granitos, gneis, esquistos metamórficos y, en porcentajes mucho más bajos, las hematites. Mientras que las rocas graníticas y esquistosas se mantienen en proporciones estables en todas las terrazas, la proporción relativa de los componentes cuarcíticos tiende a disminuir progresivamente de las terrazas superiores hacia las inferiores ${ }^{8}$. La Plane d'en Bourgat es la que presenta un mayor incremento de los elementos cuarcíticos, lo que contrasta con la escasa presencia de granitos y gneis. Los estudios morfométricos y granulométricos han permitido confirmar las mismas condiciones de deposición sedimentaria en todos los niveles fluviales. Sin embargo, la morfometría de los cantos de cuarzo de la Butte du Four-Llabanère muestra unos índices de redondeo más elevados, y la granulometría de la Plane d'en Bourgat un predominio más destacado de los elementos gruesos -entre 0,7 y $0,8 \mathrm{~mm}$ de media- en relación al resto de formaciones -donde raramente sobrepasan los 0,3 mm-. Este hecho indicaría que el curso fluvial se fue alejando paulatinamente a partir de este momento.

\subsection{Revisión geomorfológica y geocronológica}

En el primer trabajo geológico de la serie de plataformas aluviales de los ríos Têt, Tech y Agly, Depéret (1885) reconoció tres terrazas diferentes que detectó mediante sus altitudes relativas sobre el nivel del cauce del río -terraza aI-c, de 4 a $6 \mathrm{~m}$; terraza aI-b, de 15 a $20 \mathrm{~m}$, y terraza aI-a, de 30 a 35 m-. Más tarde, Bourcart (1945) asimiló estas formaciones a un suelo residual villafranquiense -2.5-1 Ma-, cubierto por una matriz de gravas rojizas con un fuerte componente de cuarcitas y

${ }^{7}$ Ibidem.

${ }^{8}$ Collina-Girard: $o p$. cit. n. 2. cuarzos eolizados. Got (1967) diferenció la serie de terrazas en: una inferior correspondiente a la plataforma caliza del Agly; una media constituida por afloramientos postvillafranquienses de la ribera izquierda del Têt, y una última superior asimilable a la definida previamente por Borcart. También Barrière (1969) confirmó la presencia de estas tres terrazas a partir del estudio de las alteraciones de las rocas que le permitieron diferenciar: los suelos de los niveles inferiores -terraza baja del Agly-, con sedimentos ligeramente depuestos en forma de colada; los de los niveles medios, con componentes sedimentarios marrones y poco alterados, y finalmente los de los niveles superiores, con sedimentos rojizos evolucionados y con rocas altamente afectadas por la alteración. El primer geólogo que ofreció una primera secuenciación cronológica fue Monaco ${ }^{9}$, quien propuso en paralelismo con los suelos descritos en los Pirineos centrales una datación villafranquiense para los niveles de aluviones rojizos superiores, mindeliana (ca. $580 \mathrm{ka})$ para los aluviones menos evolucionados medios y rissiana -c. $200 \mathrm{ka}$ - para los aluviones calizos inferiores. Según este geólogo, la formación de estas tres unidades geomorfológicas estaría relacionada con un enfriamiento climático progresivo en el que se sucedieron tres episodios principales de deposición sedimentaria y de alteración pedogenética.

El descubrimiento de un importante registro lítico sobre estas formaciones fluviales permitió a de Lumley (1969a, 1969b, 1971; de Lumley et al., 1976) establecer una relación entre el sistema de terrazas y la secuenciación cronológica relativa, que ya se había dado a conocer previamente en los primeros estudios geológicos con la obtenida a partir de los estudios tecnotipológicos de las industrias. A partir de esa correlación de datos, este investigador agrupó inicialmente para la plataforma aluvial superior la terraza de Mata Roudoume, le Correch y Mas d'Estrades; para la media la de Manadeil y Llabanère -atribuidas por sus industrias al Günz; c. 1.1 Ma-, y para la inferior la de Rivesaltes y Pia -adscritas al Mindel; $c$. $580 \mathrm{ka}-$. Sin embargo, la presencia de otras terrazas intermedias y secundarias condujo a CollinaGirard (1976a, 1976c) ${ }^{10}$ a establecer un esquema

${ }^{9}$ Cf. Monaco: op. cit. n. 3.

${ }^{10} C f$. también $o p$. cit. n. 2. 


\begin{tabular}{|c|c|c|c|c|c|c|c|c|c|c|c|c|c|}
\hline Enclaves & 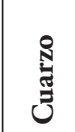 & 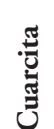 & 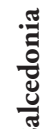 & ڤ్ & & & Enclaves & 芯 & 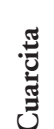 & 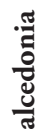 & క్ఝ & & త్ర \\
\hline Auberge du Lac Aude & - & - & - & 1 & 1 & 0,2 & Mas Rombau & 38 & 1 & - & 1 & 40 & 3,7 \\
\hline Baixades & 2 & - & - & - & 2 & 0,3 & Mas Romeu & 9 & 1 & - & 1 & 11 & 1,0 \\
\hline Butte 124 & 3 & - & - & - & 3 & 0,3 & Mas Sabole & 5 & - & - & - & 5 & 0,5 \\
\hline Butte 69 & 3 & - & - & - & 3 & 0,3 & Mas Sante Thérèse & 1 & - & - & - & 1 & 0,1 \\
\hline Butte des Estagnets & 3 & - & 2 & - & 5 & 0,8 & Mata Roudounne & 3 & - & - & - & 3 & 0,3 \\
\hline Butte du Four & 34 & 1 & 1 & - & 36 & 5,8 & Moulin du Souffre & 3 & - & - & - & 3 & 0,3 \\
\hline Cabane Auriol & 12 & - & - & - & 12 & 1,9 & Oums & 18 & - & - & 4 & 22 & 2,1 \\
\hline Calce Cabanac & - & - & - & 1 & 1 & 0,2 & Palau del Vidre & 1 & - & - & - & 1 & 0,1 \\
\hline Call d'Ayroles & 3 & - & - & - & 3 & 0,3 & Route de Pia & 7 & - & - & 1 & 8 & 0,7 \\
\hline Canohès & 6 & - & - & - & 6 & 1,0 & Pézilla de la Rivière & 7 & - & - & - & 7 & 0,6 \\
\hline Casot Azaïs & 4 & - & - & - & 4 & 0,6 & Pia Route de Bompas & 2 & - & - & - & 2 & 0,2 \\
\hline Cimetière & 2 & - & - & - & 2 & 0,3 & Pla del Rey & 27 & - & - & - & 27 & 2,5 \\
\hline Cimetière d'Espira & - & - & - & 1 & 1 & 0,2 & Plaine du Roussillon & 1 & - & - & - & 1 & 0,1 \\
\hline Claira Mas Bordas & 1 & - & - & - & 1 & 0,2 & Plane d'en Bourgat & 1 & - & - & - & 1 & 0,1 \\
\hline Coll de la Guille & 62 & - & - & - & 62 & 9,9 & Plateau & 3 & - & - & - & 3 & 0,3 \\
\hline Cyprien & 20 & 9 & 2 & 11 & 42 & 6,8 & Pollestres & 2 & - & - & - & 2 & 0,2 \\
\hline Espira de l'Agly & 16 & - & - & - & 16 & 2,6 & Porte d'Espagne & 1 & - & - & - & 1 & 0,1 \\
\hline Font de la Gas & 2 & - & - & - & 2 & 0,3 & Près des Michel & 2 & - & - & - & 2 & 0,2 \\
\hline Gourg & 20 & 1 & 4 & - & 25 & 4,0 & Puech de la Boule & 1 & - & - & - & 1 & 0,1 \\
\hline Ille-sur-Têt & 5 & - & - & - & 5 & 0,8 & Rabin de la Combe & - & - & - & 1 & 1 & 0,1 \\
\hline Jas Muscatere & 42 & - & - & - & 42 & 6,7 & Réservoir & 4 & - & - & - & 4 & 0,4 \\
\hline La Coume & 2 & - & - & - & 2 & 0,3 & Retenue Superieure & 2 & - & 1 & - & 3 & 0,3 \\
\hline La Garrigue & 1 & - & - & - & 1 & 0,1 & Rive Droite du Reart & 1 & - & - & - & 1 & 0,1 \\
\hline La Llabanère & 56 & - & - & - & 56 & 9,0 & Rive Gauche du Reart & 1 & - & - & - & 1 & 0,1 \\
\hline La Mouillere & 4 & - & - & - & 4 & 0,6 & Rivesaltes le Jas & 8 & - & - & - & 8 & 0,8 \\
\hline La Prunette & 5 & - & - & - & 5 & 0,8 & Rivesaltes Muscatère & 31 & - & - & - & 31 & 2,9 \\
\hline La Resclose & 1 & 1 & - & - & 2 & 0,3 & Rivesaltes Paniçó & - & - & - & 1 & 1 & 0,1 \\
\hline La Sabilane & 1 & - & - & - & 1 & 0,2 & Rivesaltes Saint Bernard & 1 & - & - & - & 1 & 0,1 \\
\hline La Solana & 1 & - & - & - & 1 & 0,2 & RN 117 Reservoir & 3 & - & - & - & 3 & 0,3 \\
\hline La Torra & 3 & - & - & - & 3 & 0,3 & Rodes & 569 & 16 & 5 & 17 & 607 & 57,1 \\
\hline Les Baguères & 8 & - & - & - & 8 & 1,3 & Roquessels & 54 & 12 & - & 12 & 78 & 7,3 \\
\hline Le Boulou & 8 & 1 & - & - & 9 & 1,4 & Route de Saint Estève & 1 & - & - & - & 1 & 0,1 \\
\hline Le Correch & 1 & - & - & - & 1 & 0,2 & Route de Vinça & 2 & - & - & - & 2 & 0,2 \\
\hline Les Ambuillas & 3 & - & - & - & 3 & 0,3 & Route National d'Espira & - & - & - & 1 & 1 & 0,1 \\
\hline Les Caganillas & 1 & - & - & 1 & 2 & 0,3 & Saint Jean Doserons & 3 & - & - & - & 3 & 0,3 \\
\hline Les Mirabels & 1 & - & - & - & 1 & 0,2 & Saint Michel & 1 & - & - & - & 1 & 0,1 \\
\hline Les Tuileries & 13 & - & - & - & 13 & 2,2 & Saint Pierre & 2 & - & - & - & 2 & 0,2 \\
\hline Llupia M. Saint Sauveur & 1 & - & - & - & 1 & 0,2 & Saleilles & 2 & - & - & - & 2 & 0,2 \\
\hline L'Ouliberde & 4 & - & - & - & 4 & 0,6 & Salses Mas Burguére & 1 & - & - & - & 1 & 0,1 \\
\hline Manadeil & 17 & - & - & - & 17 & 2,7 & Santa Eugénie & 1 & - & - & - & 1 & 0,1 \\
\hline Manadeil Relais PTT & 6 & - & - & - & 6 & 1,0 & Ter de la Têt la Baillobère & 3 & - & - & - & 3 & 0,3 \\
\hline Mas Bruno & 22 & 2 & - & 1 & 25 & 4,0 & Ternére & \begin{tabular}{|l|}
62 \\
\end{tabular} & - & - & - & 62 & 5,8 \\
\hline Mas Canterrene & 1 & - & - & - & 1 & 0,2 & Terrasse de Lempobe & 9 & - & 1 & 1 & 11 & 1,0 \\
\hline Mas de la Prades Bages & 13 & 1 & 3 & - & 17 & 2,7 & Terrasse du Boulou & 1 & - & - & - & 1 & 0,1 \\
\hline Mas du Crest & 5 & - & - & 3 & 8 & 1,3 & Torrella & 7 & - & - & - & 7 & 0,7 \\
\hline Mas du Romarin & 1 & - & - & - & 1 & 0,2 & Traverse de Palau & 2 & - & - & - & 2 & 0,2 \\
\hline Mas Ducup & 2 & - & - & - & 2 & 0,3 & Trouillas & 10 & - & - & - & 10 & 0,9 \\
\hline Mas Ferréol & 125 & - & - & 2 & 127 & 19,9 & Villefranche P. Ambullas & 10 & - & - & - & 10 & 0,9 \\
\hline Mas Ferrer & 14 & - & - & - & 14 & 2,3 & Villeneuve-la-Raho & 7 & - & - & - & 7 & 0,7 \\
\hline Mas Galté & 13 & - & - & 3 & 16 & 2,6 & Vinça & 21 & - & - & - & 21 & 1,9 \\
\hline Mas Lloubes & 5 & - & - & - & 5 & 0,8 & Vinça Saint Pierre & 32 & - & 3 & 1 & 36 & 3,3 \\
\hline \multirow{2}{*}{ Total } & 578 & 16 & 12 & 24 & \multirow{2}{*}{\multicolumn{2}{|c|}{630}} & \multirow{2}{*}{ Total } & 983 & 30 & 10 & 41 & \multirow{2}{*}{\multicolumn{2}{|c|}{1064}} \\
\hline & 91,7 & 2,5 & 1,9 & 3,9 & & & & 92,5 & 2,8 & 0,9 & 3,8 & & \\
\hline
\end{tabular}

FIG. 3. Enclaves de las terrazas del Rosellón según las materias primas talladas (Llupia M. Saint Sauveur = Llupia Mas Saint Sauveur). 
más detallado del sistema fluvial de la cuenca del río Têt que fue adoptado más tarde por Salvayre (1983). Este autor consideraba que la serie aluvial comenzaría con los niveles más altos y antiguos, a los que pertenecerían las terrazas de Mata Roudoume y Mas Ferréol; continuaría con los inmediatamente inferiores, como la terraza de Cabestany; los medios, con las de la Butte du Four, Llabanère y Pia; los bajos, con la serie de las de Rivesaltes y de Ille-sur-Têt, y finalmente con las más recientes e inferiores, que comprenderían las de Prades y los niveles de la llanura por la que discurre actualmente el río Têt.

Más adelante, geólogos como Clauzon (1984), Calvet $^{11}$, Giret (1995), Debals ${ }^{12}$ o Duvail et al. (2001), entre otros, han contribuido a actualizar y corregir el esquema geológico y cronológico propuesto en estos primeros estudios (Fig. 5). Gracias a la valoración conjunta de los depósitos sedimentarios aluviales desde el punto de vista de su alteración, petrografía, altitud, eolización, deformación, granulometría y tecnología lítica de los artefactos recuperados, Calvet pudo definir un nuevo modelo de evolución geocronológica de los diferentes niveles de terraza y, frente a los planteamientos de de Lumley y Collina-Girard, rejuveneció las dataciones relativas del complejo de terrazas bajas -T1 y T2- y medianas -T3-. Además, pasó a considerar los niveles fluviales de la Butte du Four, Llabanère y Pia como una misma unidad geoestratigráfica, sobre la que actuaron las deformaciones neotectónicas, lo que indujo a error a de Lumley y Collina-Girard. A su vez, agrupó los niveles de Mata Roudoume y Mas Ferréol como una única terraza -T5-, que justificó en base a los fenómenos de desplazamiento provocados por la falla que afectó a las formaciones pliocenas de Els Orgues, en Ille-sur-Têt.

Las hipótesis planteadas por Calvet sobre la neotectónica fueron corroboradas por Giret (1995) a partir del estudio morfométrico de los niveles fluviales mejor conservados y extensos del Têt, gracias a las variaciones altimétricas que detectó en los perfiles longitudinales de las terrazas. También destacó la influencia de los procesos postdeposicionales de erosión, alteración y

${ }^{11}$ Cf. Calvet: op. cit. n. 3.

${ }^{12}$ Cf. Debals: op. cit. n. 3. desplazamiento del curso fluvial, que consideró como los principales factores que permitirían explicar la dificultad para diferenciar los diversos niveles fluviales que integran el complejo de terrazas superiores y medias. Los estudios posteriores de Debals ${ }^{13}$ volvieron a ratificar el modelo que Calvet ya había expuesto, pero envejeciendo la cronología propuesta para la terraza de Thuir -T2 de Calvet-, que atribuyó a la época rissiana. Asimismo modificó la terminología propuesta por de Lumley y Collina-Girard para la terraza de Cabestany, que Debals pasó a llamar Plane d'en Bourgat. En este artículo hemos optado por adoptar esta nueva nomenclatura propuesta por este investigador en sustitución de la de Cabestany.

El sistema de terrazas del Agly, en contraposición con el del Têt, aún no ha sido objeto de estudios geológicos suficientemente profundos, ni de prospecciones arqueológicas sistemáticas que permitan contextualizar con más rigor sus industrias. Solo es posible destacar las aportaciones de Milor ${ }^{14}$, que diferenció tres terrazas fluviales -alta, media y baja- y tres conos aluviales - de Villelongue dels Monts, de Soréde y de la Massane-. Más actualmente, Debals ${ }^{15}$ ha ampliado las tesis de Milor, distinguiendo un sistema de terrazas más amplio en el que diferenció un complejo superior -terrazas de Banyuls dels Aspres superior e inferior-, medio -Les Basses y Camp de la Garrigue, aguas arriba de Le Boulou, y Can Partère, aguas abajo- e inferior -Le Boulou, por un lado, y Céret y Amélie les Bains, por otro-.

Según Clauzon (1984) y Duvail et al. (2001), Mas Ferréol se habría formado en el Günz, mientras que Cabestany -para Debals y nosotros Plane d'en Bourgat- pertenecería al Mindel. Estos estudios han permitido identificar la Butte du Four y la Llabanère como una entidad geológica rissiana diferente a la de Pia, que se habría creado después, probablemente en el Würm (c. $80 \mathrm{ka})$. Para llevar a cabo nuestro estudio tecnológico nos hemos guiado por estos planteamientos más

13 Ibidem.

${ }^{14}$ Milor, F. (1991): Les formations alluviales quaternaires de la valée du Tech (Roussillon). Memoria de Diplôme d'Études Approfondies inédita, presentada en 1991 en el Muséum National d'Histoire Naturelle de París.

${ }^{15}$ Debals: op. cit. n. 3. 


\begin{tabular}{|c|c|c|c|c|c|c|c|c|c|c|c|c|c|c|c|c|c|}
\hline Enclaves & ن. & $\frac{\dot{0}}{\tilde{D}}$ & $\begin{array}{l}\dot{ن} \\
\dot{\dot{z}} \\
\dot{z}\end{array}$ & ن & 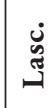 & 当 & & & Enclaves & 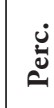 & $\frac{ن}{ن}$ & ن & 它 & نू & 宓 & & 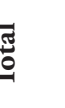 \\
\hline Auberge du Lac Aude & 1 & - & - & - & - & - & 1 & 0,1 & Mas Rombau & - & 1 & 24 & 3 & 7 & 5 & 40 & 3,8 \\
\hline Baixades & - & - & 1 & 1 & - & - & 2 & 0,3 & Mas Romeu & - & 1 & 8 & - & 2 & - & 11 & 1,0 \\
\hline Butte 124 & - & - & 3 & - & - & - & 3 & 0,4 & Mas Sabole & - & - & 5 & - & - & - & 5 & 0,5 \\
\hline Butte 69 & - & - & 3 & - & - & - & 3 & 0,4 & Mas Sante Thérèse & - & - & 1 & - & - & - & 1 & 0,1 \\
\hline Butte des Estagnets & - & 2 & 1 & 1 & 1 & - & 5 & 0,8 & Mata Roudounne & - & 1 & 2 & - & - & - & 3 & 0,3 \\
\hline Butte du Four & 1 & 3 & 18 & 3 & 4 & 7 & 36 & 5,9 & Moulin du Souffre & - & - & 3 & - & - & - & 3 & 0,3 \\
\hline Cabane Auriol & - & - & 11 & - & - & 1 & 12 & 1,9 & Oums & - & - & 16 & - & - & 6 & 22 & 2,1 \\
\hline Calce Cabanac & - & - & - & 1 & - & - & 1 & 0,1 & Palau del Vidre & - & - & 1 & - & - & - & 1 & 0,1 \\
\hline Call d'Ayroles & - & - & 1 & - & 2 & - & 3 & 0,4 & Route de Pia & - & - & 6 & 1 & - & 1 & 8 & 0,7 \\
\hline Canohès & - & - & 6 & - & - & - & 6 & 0,9 & Pézilla de la Rivière & - & 1 & 6 & - & - & - & 7 & 0,6 \\
\hline Casot Azaïs & 1 & - & 2 & - & 1 & - & 4 & 0,6 & Pia Route de Bompas & - & - & 2 & - & - & - & 2 & 0,2 \\
\hline Cimetière & - & - & 1 & - & - & 1 & 2 & 0,3 & Pla del Rey & - & 2 & 23 & - & - & 2 & 27 & 2,7 \\
\hline Cimetière d'Espira & - & - & - & - & - & 1 & 1 & 0,1 & Plaine du Roussillon & - & - & 1 & - & - & - & 1 & 0,1 \\
\hline Claira Mas Bordas & - & - & 1 & - & - & - & 1 & 0,1 & Plane d'en Bourgat & - & - & 1 & - & - & - & 1 & 0,1 \\
\hline Coll de la Guille & 2 & 3 & 38 & 4 & 8 & 7 & 62 & 10,1 & Plateau & - & - & 3 & - & - & - & 3 & 0,3 \\
\hline Cyprien & - & 13 & 11 & 1 & 8 & 9 & 42 & 6,9 & Pollestres & - & - & 1 & 1 & - & - & 2 & 0,2 \\
\hline Espira de l'Agly & 5 & 2 & 4 & - & 1 & 4 & 16 & 2,6 & Porte d'Espagne & - & - & - & - & - & 1 & 1 & 0,1 \\
\hline Font de la Gas & - & - & 1 & - & - & 1 & 2 & 0,3 & Près des Michel & - & - & 1 & 1 & - & - & 2 & 0,2 \\
\hline Gourg & 2 & 7 & 6 & - & - & 10 & 25 & 4,1 & Puech de la Boule & - & - & - & - & - & 1 & 1 & 0,1 \\
\hline Ille-sur-Têt & 1 & - & 4 & - & - & - & 5 & 0,8 & Rabin de la Combe & - & - & - & - & - & 1 & 1 & 0,1 \\
\hline Jas Muscatère & - & - & 35 & 6 & 1 & - & 42 & 6,8 & Réservoir & - & - & 3 & - & - & 1 & 4 & 0,4 \\
\hline La Coume & - & - & 1 & - & 1 & - & 2 & 0,3 & Retenue Superieure & - & - & 2 & - & - & 1 & 3 & 0,3 \\
\hline La Garrigue & - & - & - & - & - & 1 & 1 & 0,1 & Rive Droite du Reart & - & - & 1 & - & - & - & 1 & 0,1 \\
\hline La Llabanère & 1 & 14 & 32 & 3 & - & 6 & 56 & 9,0 & Rive Gauche du Reart & - & - & 1 & - & - & - & 1 & 0,1 \\
\hline La Mouillere & - & 1 & 3 & - & - & - & 4 & 0,6 & Rivesaltes le Jas & - & 1 & 6 & - & 1 & - & 8 & 0,7 \\
\hline La Prunette & - & 1 & - & - & 4 & - & 5 & 0,8 & Rivesaltes Muscatère & - & 4 & 19 & - & 4 & 4 & 31 & 2,9 \\
\hline La Resclose & - & - & 2 & - & - & - & 2 & 0,3 & Rivesaltes Paniçó & - & - & 1 & - & - & - & 1 & 0,1 \\
\hline La Sabilane & - & 1 & - & - & - & - & 1 & 0,1 & Rivesaltes Saint Bernard & - & - & - & - & - & 1 & 1 & 0,1 \\
\hline La Solana & - & - & 1 & - & - & - & 1 & 0,1 & RN 117 Reservoir & - & - & 3 & - & - & - & 3 & 0,3 \\
\hline La Torra & - & - & 2 & - & - & 1 & 3 & 0,4 & Rodes & - & 3 & 181 & 11 & 314 & 98 & 607 & 56,9 \\
\hline Les Baguères & 1 & - & 3 & 1 & 2 & 1 & 8 & 1,3 & Roquessels & 1 & 10 & 19 & 6 & 6 & 36 & 78 & 7,3 \\
\hline Le Boulou & - & - & 4 & 3 & 1 & 1 & 9 & 1,4 & Route de Saint Estéve & - & - & 1 & - & - & - & 1 & 0,1 \\
\hline Le Correch & - & - & 1 & - & - & - & 1 & 0,1 & Route de Vinça & - & - & 1 & - & - & 1 & 2 & 0,2 \\
\hline Les Ambuillas & - & - & 3 & - & - & - & 3 & 0,4 & Route National d'Espira & - & 1 & - & - & - & - & 1 & 0,1 \\
\hline Les Caganillas & - & 1 & - & - & - & 1 & 2 & 0,3 & Saint Jean Doserons & - & - & 3 & - & - & - & 3 & 0,3 \\
\hline Les Mirabels & - & - & 1 & - & - & - & 1 & 0,1 & Saint Michel & - & - & 1 & - & - & - & 1 & 0,1 \\
\hline Les Tuileries & - & - & 10 & - & - & 3 & 13 & 2,2 & Saint Pierre & - & - & 2 & - & - & - & 2 & 0,2 \\
\hline Llupia M. S. Sauveur & - & - & 1 & - & - & - & 1 & 0,1 & Saleilles & - & - & 2 & - & - & - & 2 & 0,2 \\
\hline L'Ouliberde & - & 4 & - & - & - & - & 4 & 0,6 & Salses Mas Burguére & - & - & 1 & - & - & - & 1 & 0,1 \\
\hline Manadeil & 1 & 2 & 13 & - & - & 1 & 17 & 2,8 & Santa Eugénie & - & - & 1 & - & - & - & 1 & 0,1 \\
\hline Manadeil Relais PTT & - & - & 6 & - & - & - & 6 & 1,0 & Ter de la Têt la Baillobère & - & - & 1 & - & - & 2 & 3 & 0,3 \\
\hline Mas Bruno & - & - & 21 & - & 2 & 2 & 25 & 4,2 & Ternére & - & 2 & 49 & - & 8 & 3 & 62 & 5,8 \\
\hline Mas Canterrene & - & 1 & - & - & - & - & 1 & 0,1 & Terrasse de Lempobe & 1 & 1 & 4 & - & 3 & 2 & 11 & 1,0 \\
\hline Mas de la P. Bages & - & - & 15 & 1 & 1 & - & 17 & 2,9 & Terrasse du Boulou & - & - & - & - & - & 1 & 1 & 0,1 \\
\hline Mas du Crest & - & 1 & 7 & - & - & - & 8 & 1,4 & Torrella & - & 1 & 5 & - & 1 & - & 7 & 0,6 \\
\hline Mas du Romarin & - & - & - & - & - & 1 & 1 & 0,1 & Traverse de Palau & - & - & 2 & - & - & - & 2 & 0,2 \\
\hline Mas Ducup & - & 1 & 1 & - & - & - & 2 & 0,4 & Trouillas & - & - & 8 & - & 1 & 1 & 10 & 0,9 \\
\hline Mas Ferréol & 1 & 21 & 61 & - & 6 & 33 & 122 & 19,1 & Villefranche P. Ambullas & - & - & 10 & - & - & - & 10 & 0,9 \\
\hline Mas Ferrer & - & 1 & 10 & - & 2 & 1 & 14 & 2,3 & Villeneuve-la-Raho & 1 & - & 4 & - & - & 2 & 7 & 0,6 \\
\hline Mas Galté & 2 & 2 & 9 & - & 1 & 2 & 16 & 2,7 & Vinça & - & 1 & 19 & - & - & 1 & 21 & 2,0 \\
\hline Mas Lloubes & - & 1 & 2 & - & 1 & 1 & 5 & 0,9 & Vinça Saint Pierre & - & - & 25 & 1 & 5 & 5 & 36 & 3,4 \\
\hline \multirow{2}{*}{ Total } & 19 & 82 & 356 & 25 & 47 & 96 & \multirow{2}{*}{\multicolumn{2}{|c|}{625}} & \multirow{2}{*}{ Total } & 3 & 30 & 479 & 24 & 352 & 176 & \multirow{2}{*}{\multicolumn{2}{|c|}{1064}} \\
\hline & 3,0 & 12,9 & 56,9 & 4,0 & 7,5 & 15,7 & & & & 0,3 & 2,8 & 45,0 & 2,2 & 33,2 & 16,5 & & \\
\hline
\end{tabular}

FIG. 4. Relación de los enclaves de las terrazas del Rosellón según los tipos de objetos técnicos (Llupia M. S. Sauveur = Llupia Mas Saint Sauveur; Mas de la P. Bages = Mas de la Prades Bages; Perc. = Percutores; Útil c. = Útiles sobre canto; Núc. $c .=$ Núcleos sobre canto; Núc. l. $=$ Núcleos sobre lasca; Lasc. $=$ Lascas y Útil. l. $=$ Útiles sobre lasca). 


\begin{tabular}{|c|c|c|c|c|}
\hline & \multicolumn{2}{|c|}{$\begin{array}{l}\text { de Lumley et al., 1976; } \\
\text { Collina-Girard, 1975 }\end{array}$} & \multicolumn{2}{|r|}{ Calvet, 1994} \\
\hline & Nivel & Cronologia & Nivel & Cronología \\
\hline \multirow{3}{*}{ Terrazas altas } & Mata Roudoume & \multirow{3}{*}{ Günz (1.1 Ma) } & T5 & \multirow{3}{*}{ Villafranquiense (2.5-1 Ma } \\
\hline & Mas Ferréol & & (1) & \\
\hline & \begin{tabular}{|l|} 
Cabestany \\
\end{tabular} & & T4 & \\
\hline \multirow[t]{3}{*}{ Terrazas medias } & Butte du Four & \multirow{3}{*}{ Mindel $(c .580 \mathrm{ka})$} & \multirow{3}{*}{ T3 } & \multirow{3}{*}{ Riss (c. $200 \mathrm{ka})$} \\
\hline & Llabanère & & & \\
\hline & Pia & & & \\
\hline \multirow{2}{*}{ Terrazas bajas } & Ille-sur-Têt & Riss (c. $200 \mathrm{ka})$ & T2 & \multirow{2}{*}{ Würm $(c .80 \mathrm{ka})$} \\
\hline & Prades & Würm $(c .80 \mathrm{ka})$ & T1 & \\
\hline \multirow{4}{*}{ Aluviones actuales } & Lecho mayor & \multirow{2}{*}{ Actual } & T0 & \multirow{2}{*}{ Actual } \\
\hline & Lecho menor & & & \\
\hline & \multicolumn{2}{|c|}{ Debals, 1998} & \multicolumn{2}{|r|}{$\begin{array}{c}\text { Clauzon, 1984; } \\
\text { Duvail et al., 2001 }\end{array}$} \\
\hline & Nivel & Cronología & Nivel & Cronología \\
\hline \multirow{2}{*}{ Terrazas altas } & Mas Ferréol & $1070-990 \mathrm{ka}$ & Fw & Günz (1.1 Ma) \\
\hline & Plane d'en Bourgat & $900-700 \mathrm{ka}$ & Fxa-Fx1 & Mindel $(c .580 \mathrm{ka})$ \\
\hline \multirow{3}{*}{ Terrazas medias } & Butte du Four & \multirow{3}{*}{$<780 \mathrm{ka}$} & Fxb1 & \multirow{2}{*}{ Riss (c. $200 \mathrm{ka})$} \\
\hline & Llabanère & & Fxb1-Fx2 & \\
\hline & Pia & & Fyb-Fy1 & \multirow{2}{*}{ Würm (c. $80 \mathrm{ka})$} \\
\hline \multirow{2}{*}{ Terrazas bajas } & Thuir & Riss (c. $200 \mathrm{ka})$ & Fya-Fy1 & \\
\hline & Ille-sur-Têt & Würm $(c .80 \mathrm{ka})$ & Fy2 & Tardiglaciar (c. 13-10 ka) \\
\hline Aluviones actuales & Llanura aluvial & Actual & $\begin{array}{l}\text { Fz1 } \\
\text { Fz2 }\end{array}$ & Actual \\
\hline
\end{tabular}

FIG. 5. Comparación de las estimaciones geoestratigráficas y cronológicas para el sistema de terrazas del Rosellón.

actuales, de forma que hemos optado por no incluir las industrias de Pia en el complejo de las terrazas medias -terraza de la Butte du Four-Llabanère-, ya que pertenecen a épocas que superan nuestro ámbito cronológico de estudio. En todo caso, tal y como nuestros análisis tecnológicos sugieren, las cronologías de las industrias localizadas sobre estas superficies fluviales coincidirían con las edades sugeridas por Clauzon (1984) y Duvail et al. (2001) (Tabla 3). Por lo tanto, esto implicaría que las ocupaciones humanas se establecieron en las terrazas en épocas no muy posteriores a su edad de formación.

\section{Correlación geocronológica de las terrazas con la industria lítica}

Con el objetivo de correlacionar las industrias de estas terrazas fluviales con su evolución geológica y de obtener cronologías relativas de las ocupaciones humanas, hemos seguido los esquemas geocronológicos propuestos en los trabajos sobre los registros líticos llevados a cabo por de Lumley $(1969 a, 1969 b, 1971$; de Lumley et al., 1976) y Collina-Girard (1976a, $1976 c)^{16}$, y de las formaciones fluviales cuaternarias de Clauzon (1984), Calvet ${ }^{17}$, Giret (1995), Debals ${ }^{18}$ y Duvail et al. (2001). Como todos ellos apuntan, la asociación de las industrias a la formación del sistema de terrazas del Rosellón permite afirmar que ambos fenómenos pudieron tener lugar entre el Villafranquiense o el Günz y los inicios del Mindel (Fig. 5).

En un primer momento, durante las últimas fases del Plioceno, es decir, a finales de este límite cronológico superior, se depusieron en el sustrato del Têt niveles de arcillas marinas. Paralelamente, se produjo el desmantelamiento de los macizos graníticos y esquistosos, junto con la formación de depósitos arcósicos y de coluviones arcillo-esquistosos rojizos. Con posterioridad habría tenido lugar una regresión marina en la que las formaciones de origen marino habrían sido reemplazadas por depósitos sedimentarios fluviales continentales de tipo torrencial. Es esta segunda fase sedimentaria, adscribible al Günz según la mayoría de autores -aunque Calvet plantea que se produjo en épocas villafranquienses-, la primera que podemos correlacionar de manera directa con las primeras ocupaciones humanas del Rosellón, que se habrían establecido sobre los niveles de la terraza de Mas Ferréol. En esos momentos habría tenido lugar un importante episodio erosivo, que acabó

${ }^{16}$ Cf. también op. cit. n. 2.

${ }^{17}$ Cf. op. cit. n. 2.

18 Debals: op. cit. n. 3. 
con la deposición en el Pleistoceno inferior de este depósito de origen fluvio-torrencial sobrepuesto directamente sobre las últimas formaciones pliocenas.

Lamentablemente, las dataciones paleomagnéticas de Debals ${ }^{19}$ sobre los niveles de Mas Ferréol no han proporcionado resultados concluyentes, aunque se han intentado correlacionar con la terraza superior del Verdouble, en el valle de Tautavel. Para esta formación, Laaraich ${ }^{20}$ obtuvo una cronología de $930 \pm 130$ ka a partir de una muestra de gravas de cuarzo por la técnica del ESR y a partir de la aplicación del paleomagnetismo una polaridad positiva. Los aluviones superiores del Verdouble se situarían dentro del cron Matuyama, en el episodio positivo del Jaramillo -1.07-0.99 Ma-, siendo esta misma cronología la que Debals extrapoló para Mas Ferréol. Al mismo tiempo, correlacionó el momento de la deposición de los sedimentos de Mas Ferréol con las cronologías obtenidas en otras terrazas antiguas situadas en regiones cercanas. Sería el caso de los 1.5 Ma obtenidos por Ambert (1993) para el nivel fluvial superior de Lézignan la Cébe y por Barriére y Michaux (1973) para el del Hérault, cerca de Pézenas, en Valros, de los que más tarde Sémah (1977) obtuvo un intervalo entre 1.4 y $1.6 \mathrm{Ma}$, que corroboraron las tesis iniciales de Barriére y Michaux (Figs. 3-5).

Posteriormente a la deposición de Mas Ferréol, la actuación de una nueva fase erosiva en la llanura del Rosellón provocó el encajamiento de una nueva red hidrográfica. Seguramente esto tuvo lugar a finales del

${ }^{19}$ Debals: op. cit. n. 3.

${ }^{20}$ Laaraich, M.: Les formations superficielles de la moyenne vallée de l'Agly et son bassin versant (Maury et le Verdouble) Roussillon, Pyrénées Orientales. Tesis doctoral inédita, presentada en 1996 en el Muséum National d'Histoire Naturelle de Paris.
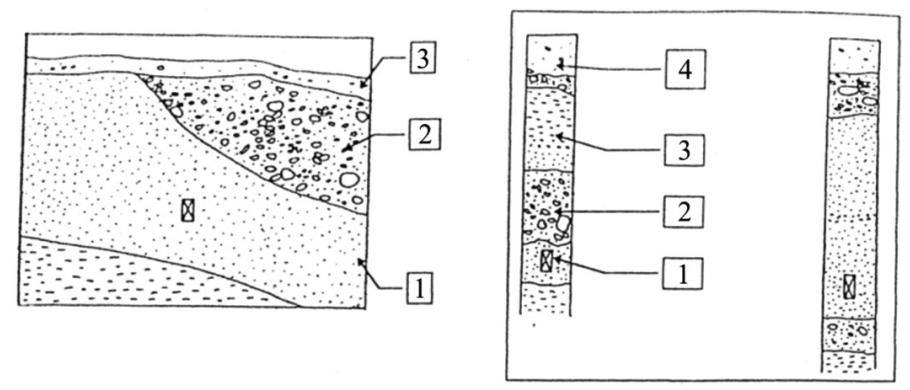

Alleaux

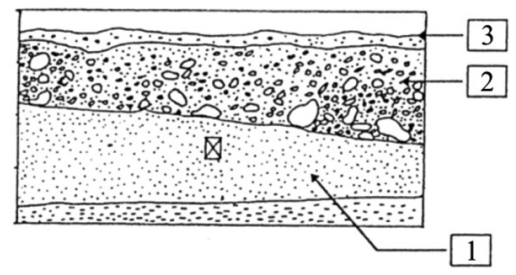

Done Morte

Escala: $1 \mathrm{~cm}=50 \mathrm{~cm}$

Nivel de arenas $\cdots$ Nivel de gravas $\because$ Nivel de limos

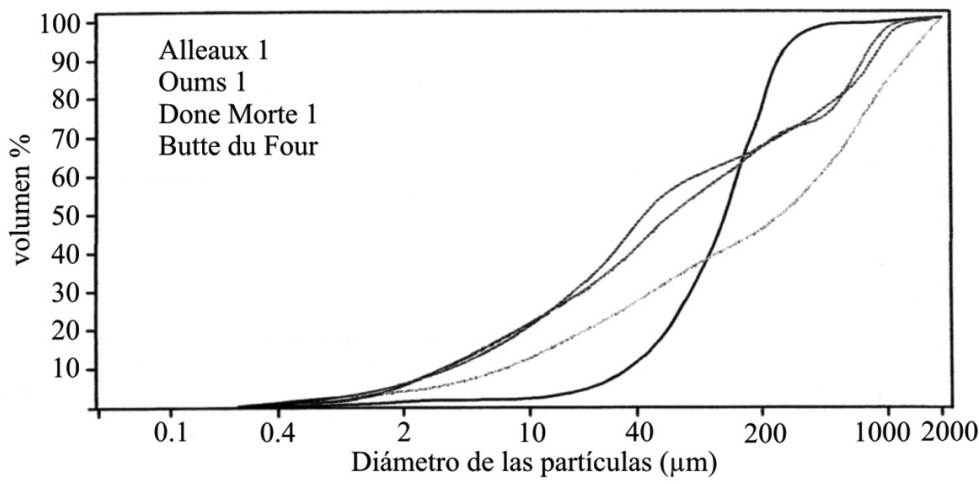

FIG. 6. Situación de las muestras extraidas de los niveles fluviales superiores y medios para la realización de los análisis paleomagnéticos y curvas granulométricas de los sedimentos (adaptación de Debals, op. cit. n. 3: 136 y 138).

momento de la formación de esta terraza superior y permitió la sedimentación de la extensa llanura aluvial de la Plane d'en Bourgat. La confluencia de diversos torrentes cerca de la localidad de Millas aportó a la terraza grandes cantos de cuarzo procedentes del desmantelamiento de los macizos graníticos, que pudieron ser aprovechados 

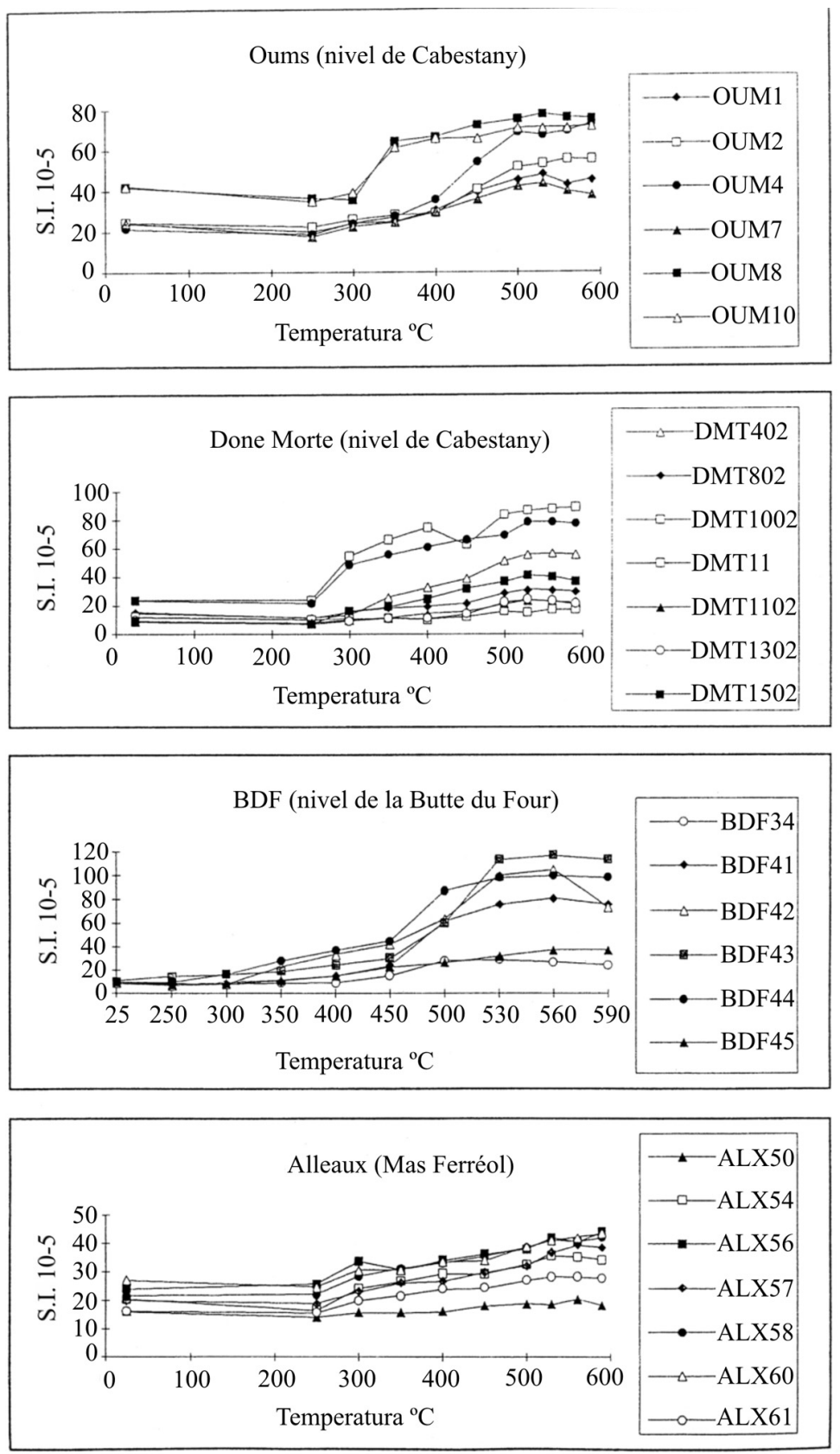

FIG. 7. Evolución de la susceptibilidad magnética de los niveles fluviales superiores y medios en función de la temperatura (adaptación de Debals, op. cit. n. 3: 146).

para la talla por las comunidades homínidas. Estas primeras ocupaciones establecidas en la Plane d'en Bourgat, probablemente, tuvieron lugar en el GünzMindel $-c .750 \mathrm{ka}-\mathrm{o}$ en el Mindel -c. $580 \mathrm{ka}-$.
Calvet continuó adscribiendo esta formación al Villafranquiense, mientras que Debals, basándose en estudios paleomagnéticos realizados sobre diversas muestras extraídas de las secciones estratigráficas de Oums y Done Morte, propuso una cronología relativa entre 900 y $700 \mathrm{ka}$. Sin embargo, Clauzon (1984) y Duvail et al. (2001) rejuvenecieron estas cronologías, atribuyéndola al Mindel. A juzgar por la pobreza del registro lítico recuperado, estas ocupaciones parecen haber sido dispersas y poco importantes. Hay que destacar que la superficie de estos objetos líticos está afectada por una alteración y rubefacción mucho más intensas, en comparación con las terrazas de cronologías posteriores.

A inicios del Mindel, según de Lumley et al. (1976) y Collina-Girard (1975), y del Riss, según el resto de autores, actuaron importantes fenómenos tectónicos en un contexto de continuos cambios climáticos que acabaron provocando la deformación de la estructura anticlinal de la terraza de la Plane d'en Bourgat. Paralelamente, la intensa erosión ocasionada por la acción de los cursos hídricos del Têt produjo el encajamiento y configuración definitiva de su lecho fluvial. Es en estos momentos cuando se depusieron los niveles sedimentarios que constituyen la terraza de la Butte du Four-Llabanère. Según la serie de dataciones paleomagnéticas realizada por Debal ${ }^{21}$ en el complejo de terrazas más antiguas del Têt, debería situarse en una época posterior al límite Matuyama-Brunhes, sobre los 780 ka (Figs. 3-5). La fuerte pátina que presenta el registro lítico de la Plane d'en Bourgat ha permitido evidenciar la existencia de un intenso proceso erosivo de eolización, que probablemente actuó en el periodo de formación de la Butte de Four-Llabanère.

${ }^{21}$ Debals: op. cit. n. 3. 


\section{Materiales y métodos}

Presentamos la industria lítica organizada por categorías de objetos y materias primas. Hemos procedido a su análisis siguiendo el concepto de chaîne opératoire, discriminando los aspectos tecnológicos relacionados con los procesos de configuración y explotación (Carbonell et al., 1983, 1992; Pelegrin et al., 1988; de la Torre y Mora, 2009). Los sistemas de configuración de útiles se analizan diferenciando sus potencialidades operativas -diedros, triedros y asociaciones potenciales- y su tipología -chopper, chopping-tool o bifaz en los artefactos sobre canto y raedera, denticulado, muesca, punta o bifaz en los útiles sobre lasca-. Las estrategias de explotación de los núcleos se distinguen según su facialidad - unifacial, bifacial, trifacial o multifacial-, organización de la talla -unipolar, bipolar o multipolar, distinguiendo entre las direcciones de los levantamientos opuestos, ortogonales o centrípetos- y tipología-epannelé, discoide, Levallois y poliedro-.

Estos objetos, debido a la convergencia tecnológica detectada entre algunos útiles sobre canto y núcleos, se han discriminado teniendo en cuenta la combinación de los siguientes criterios: el grado de estandarización y sistematización de los bordes configurados (Hiscock, 1997), el tamaño de las lascas extraídas de los bordes -de muy pequeño formato y regulares en el caso de los útiles-o planos de explotación -de un formato que permita su funcionalización posterior en el de los núcleos- (Kuhn, 1997) y la correspondencia morfológica con los morfotipos establecidos por la tipología. En estudios precedentes de la industria de las terrazas del Rosellón (García Garriga, 2008, 2010, $2011)^{22}$ se documenta cierta ambivalencia tecnológica o "campo operativo" (Guilbaud, 1995) entre útiles y núcleos, en parte debida al abandono recurrente de los objetos en estadios iniciales de la talla, lo que dificulta en ocasiones su discriminación según su configuración o explotación. En cualquier

$$
{ }^{22} \text { Cf. también Collina-Girard: op. cit. n. } 2 .
$$

caso, los conjuntos líticos de las tres terrazas, especialmente entre Mas Ferréol y la Plane d'en Bourgat, muestran un importante grado de homogeneidad y uniformidad tecnológica. Comparten porcentajes similares de materias primas, tipos de artefactos, tanto sobre canto como sobre lasca, y de núcleos. Este criterio tecnológico está relacionado con las conclusiones derivadas de las pátinas de la industria, que muestran el mismo nivel de erosión eólica entre el registro lítico de cada una de las tres terrazas, lo que nos permite afirmar que se trata de materiales sincrónicos y descartar posibles mezclas de materiales de épocas diversas (Fig. 9).

\section{Datos del análisis de la industria lítica}

\subsection{La terraza de Mas Ferréol}

Los asentamientos humanos de la terraza de Mas Ferréol desarrollaron de forma sistemática 
tanto secuencias de configuración de artefactos como de producción de lascas. La situación de las localidades de Mas Ferréol y Mata Roudoume es muy estratégica, emplazándose sobre una superficie ligeramente sobreelevada respecto a la llanura del Rosellón. El curso del río Têt discurría cerca de estos enclaves, lo que debió facilitar la recolección de las rocas necesarias para la talla en su lecho. Estas ocupaciones usaron masivamente el cuarzo -aparte de esta roca disponemos de un solo efectivo de granito y otro de arenisca- (Figs. 3 y 10$)$.

Los procesos técnicos de configuración de la terraza de Mas Ferréol están bien contrastados por la presencia de 21 útiles sobre canto y 33 lascas retocadas. Los soportes escogidos -lascas o cantos- para configurar los útiles muestran prácticamente la misma frecuencia de selección, con una ligera preferencia por el retoque de lascas. Los 54 artefactos documentados representan el $42,5 \%$ del registro lítico. El principal objetivo técnico de la configuración es la obtención de diedros, con algunas diferencias significativas dependiendo del tipo de soporte sobre el que se activaron. Así pues, cuando se configuraron los cantos se prefirió activar diedros no denticulados -choppers-, mientras que cuando se retocaron las lascas se tendió a elaborar denticulados. En general, la diversidad de los tipos de útiles configurados es mayor entre las lascas retocadas (Fig. 11).

La localización de los bordes configurados también es diferente, puesto que en los útiles sobre canto los diedros se sitúan, excepto en dos instrumentos, en el extremo distal de los cantos. En cambio, en las lascas retocadas, aunque los bordes distales también están presentes, los laterales también fueron importantes en la creación de diedros. Además, los útiles sobre lasca difieren respecto a los elaborados sobre canto, puesto que presentan diversos tipos de configurados asociados -4 dobles diedros denticulados recto-convexos y 1 diedro denticulado recto asociado a otro diedro cóncavo-. En cuanto a las cadenas operativas de explotación, la gran mayoría de los 61 núcleos documentados fueron objeto de una explotación con escasa organización técnica que solió implicar la extracción de lascas de forma lineal desde uno de los planos transversales del soporte. No obstante, también se llevaron a cabo gestiones más elaboradas como las bifaciales multipolares centrípetas (discoides), que sólo hemos documentado en dos núcleos (Fig. 11).

Las conclusiones tecnológicas resultantes de Mas Ferréol pueden resumirse en los siguientes puntos: 1) recolección en un área de captación inmediata de las rocas necesarias para desarrollar los procesos de talla; 2) utilización sistemática del cuarzo como roca básica en la talla -sólo se recuperaron en las prospecciones un chopper de granito y un núcleo de arenisca-; 3) importancia de las cadenas operativas de explotación; 4) las cadenas de configuración sólo están documentadas por los útiles sobre canto-choppers-; 5) las cadenas operativas de explotación muestran una diversidad más amplia, y están caracterizadas por los sistemas unifaciales y bifaciales unipolares de poca elaboración y complejidad; 6) mayor diversidad de modelos de configuración sobre las lascas -destaca la configuración de denticuladosrespecto a los artefactos sobre canto; 7) no se observa una preferencia importante en la selección diferencial de lascas o cantos para efectuar la configuración; y 8) fragmentación de la cadena operativa de configuración y explotación por la práctica ausencia de lascas, como consecuencia de la dificultad de identificación sobre el terreno, especialmente de las más pequeñas, y de la dispersión producida por los fenómenos de erosión eólica (Figs. 11 y 12).

\subsection{La terraza de la Plane d'en Bourgat}

Las ocupaciones de la Plane d'en Bourgat estuvieron especializadas en la talla del cuarzo, que permitió el desarrollo sistemático de las secuencias de configuración de instrumentos y de producción de lascas. Este material, junto con el resto -cuarcita, arenisca, corneana, calcedonia, caliza, granito, lidita, pórfido y sílex-, fue captado en zonas próximas al curso del Têt (Figs. 3 y 10). La conversión de los cantos de río en artefactos afectó a 21 elementos y el retoque de lascas a 27. Estos 48 útiles significan el 39,0\% respecto al global del registro, lo que supone un valor muy importante. La diferencia entre cantos y lascas configuradas es de tan sólo 6 efectivos más en el caso de estas últimas, de modo que cuando era 
necesario un instrumento se tallaba sin tener en cuenta el soporte.

En relación a los objetivos técnicos de las cadenas de configuración, entre los artefactos sobre canto son exclusivos los diedros -choppers-, algo que no sucede entre las lascas retocadas. No obstante, en este último caso, solo hemos contabilizado un único tipo de artefacto - una puntadiferente al diedro -raederas, denticulados y muescas-. La diversidad técnica de estos modelos es prácticamente idéntica entre los dos tipos de soporte. Además, los tipos de rocas implicadas en la configuración también son muy similares, puesto que las dos categorías de objetos comparten cinco rocas diferentes: cuarzo, cuarcita, granito y calcedonia, y arenisca, que es la única en la que varía en los artefactos sobre canto y así como el pórfido en los elaborados sobre lasca. Otra diferencia importante la encontramos en el mayor número de tipos de configuración asociados sobre el mismo soporte en las lascas retocadas, que nada más encontramos en un doble diedro -choppersobre un canto de cuarzo (Fig. 11).

En cuanto a las cadenas de explotación, los núcleos suman 58 efectivos, de los que solo uno es sobre lasca, mientras que el resto es sobre canto. Estos núcleos constituyen el 47,2\% del registro, que hace de los procesos de explotación de la Plane d'en Bourgat un objetivo técnico básico. La producción de lascas se realizó directamente sobre los cantos seleccionados, quizás por su buena disponibilidad en el entorno inmediato, de tal forma que no habría sido necesario el retoque de lascas. Numéricamente, la máxima representación de los sistemas de explotación la tienen los núcleos unifaciales y bifaciales de extracciones unipolares o lineales, seguidos de los bifaciales multipolares centropolarizados. Por consiguiente, las gestiones estuvieron básicamente dirigidas a la explotación rápida y poco elaborada de los núcleos. No obstante, también se activaron otros sistemas de explotación de mayor complejidad técnica, como los bifaciales multipolares centrípetos -discoides y Levallois-, que permitían obtener productos con bordes más potentes y afilados raramente retocados, siendo éste el caso de las lascas Levallois (Fig. 11).

De todas las consideraciones tecnológicas argumentadas sobre la industria lítica de la terraza de la Plane d'en Bourgat podemos concluir los siguientes aspectos: 1) el aprovisionamiento de las materias primas necesarias para desarrollar las secuencias técnicas se efectuó en el entorno inmediato al lugar de asentamiento; 2) existe una especialización en la utilización del cuarzo, tanto en la configuración de instrumentos como en la producción de lascas; 3) la cuarcita, arenisca, granito y calcedonia se reservaron para las cadenas de configuración, mientras que se prefirió explotar los soportes de lidita y sílex; 4) tanto en los útiles sobre canto como sobre lasca el objetivo prácticamente exclusivo de la configuración fue la activación de diedros; 5) se constata una diversidad prácticamente idéntica de los sistemas de configuración habilitados sobre canto y lasca; 6) las secuencias de explotación son generalmente poco elaboradas, con la extracción de lascas mediante gestiones unifaciales y bifaciales unipolares, pero también abundan las bifaciales multipolares centrípetas - discoides y Levallois-; 7) la diversidad técnica de los sistemas de explotación contrasta con la especialización técnica evidenciada en el único sistema de configuración sobre canto documentado -choppers-; y 8) las cadenas de configuración y explotación están bien representadas por útiles sobre canto, núcleos en sus diversas fases de explotación -dos están completamente agotados-, lascas retocadas y núcleos sobre lasca. Pese a ello, hay una carencia de objetos más pequeños, entre los cuales se encuentran especialmente las lascas, debido a la erosión eólica y a los problemas de discriminación en las prospecciones (Figs. 11 y 12).

\subsection{La terraza de la Butte du Four-Llabanère}

El complejo arqueológico en superficie de la terraza de la Butte du Four-Llabanère fue ocupado por comunidades homínidas que tuvieron en la producción de lascas el principal objetivo técnico. La presencia de 510 núcleos permite afirmar que existe un sesgo significativo en relación a las 164 lascas documentadas. La materia prima básica en la obtención de lascas es el cuarzo $(98,3 \%)$, cuya representación porcentual deja a las demás rocas en una posición muy secundaria. La captación de los materiales pudo realizarse en las mismas llanuras aluviales sobre las que estaban 
establecidas las ocupaciones de la Butte du FourLlabanère y en las cercanas riberas del río Têt (Figs. 3 y 10).

Pese a la preponderancia de la explotación, la configuración también fue importante. Así pues, la transformación de cantos y lascas en artefactos afectó a 199 elementos, lo que significa que el $22,3 \%$ de los objetos del registro fueron objeto de configuración. De todos estos instrumentos, en el $77,4 \%$ de las ocasiones se escogieron lascas para el retoque y sólo en el 22,6\% restante se seleccionaron cantos. Consecuentemente, se prefirió configurar las lascas, que presentan una mayor variedad de morfotipos de configurados. El objetivo de la configuración fue casi siempre el de activar diedros, que en los cantos-choppers y chopping-tools- están localizados en la parte distal de los soportes y en las lascas -raederas, denticulados y muescas- en los laterales. En los artefactos sobre canto se optó preferiblemente por utilizar la configuración unifacial-choppers-, mientras que en los útiles sobre lasca se prefirió la bifacial. Una nueva diferencia importante es que, cuando la configuración afectó a los cantos, los diedros se confeccionan con series de retoques continuos no denticulados, a la inversa que cuando se activaron en las lascas, en las que se prefirió recurrir a la denticulación para potenciar la capacidad operativa del filo (Fig. 11).

Aparte de los diedros simples -choppers y chopping-tools en los cantos y raederas, denticulados y muescas en las lascas retocadas-, los artefactos más significativos son los cuatro bifaces. Generalmente, tienen un aspecto masivo y presentan la parte proximal reservada o cortical, coincidiendo con el bifaz del tipo abbevilliense. Tres de ellos se configuraron sobre cantos ovalados - dos son de cuarzo y uno de caliza- y sólo uno se realizó sobre una lasca de cuarzo de gran formato. El aprovisionamiento de lascas para usarlas directamente o para reservarlas para la configuración fue el principal objetivo técnico de la Butte du Four-Llabanère. A pesar de que las lascas están infrarrepresentadas, así lo permiten confirmar los 510 núcleos, 479 de los cuales son sobre canto y 31 sobre lasca, que constituyen más de la mitad de los objetos líticos del conjunto $(57,2 \%)$. Si las sumamos a las 164 lascas y a los 154 artefactos retocados, comprobaremos que los elementos técnicos producidos mediante el desarrollo de los sistemas de explotación representan el $92,9 \%$, lo que implica que estas secuencias técnicas tuvieron un papel casi exclusivo. Raramente se tuvo que recurrir a la explotación de las lascas -aun así, los núcleos sobre lasca llegan a 31 efectivos, es decir, un 3,5\% del total-, seguramente porque la explotación de los cantos rodados, de muy fácil captación, ya debía proporcionar las lascas necesarias.

Los sistemas de explotación desarrollados son muy diversos y, al igual que en las dos terrazas superiores, los sistemas puestos en práctica reflejan un comportamiento técnico basado en una escasa complejidad. De hecho, la mayor parte de los núcleos muestran una reducción mediante extracciones unifaciales y bifaciales lineales que parten de la percusión efectuada sobre uno de los dos planos transversales del soporte. También están bien representados los sistemas unifaciales y bifaciales bipolares opuestos, bipolares y multipolarizados ortogonales y trifaciales multipolares. Estas gestiones, normalmente bien adaptadas a la morfología y dimensiones de los volúmenes y que precisaban de una escasa elaboración, contrastan con las bifaciales multipolares centrípetas y especialmente con las Levallois. Pese a existir una aleatoria selección de rocas para el desarrollo de los diversos sistemas de explotación, la presencia de un núcleo Levallois de jaspe de excelente aptitud para la talla demuestra que la calidad que ofrecían ciertos materiales fue aprovechada cuando se requerían lascas con una mayor capacidad y potencial de uso (Fig. 11).

Recapitulando, la industria de la Butte du Four-Llabanère presenta las siguientes características: 1) aprovisionamiento de las materias primas en las llanuras aluviales del Têt o en sus zonas de ribera, situadas en la propia área de influencia de los enclaves; 2) utilización sistemática del cuarzo para la configuración del utillaje y la producción de lascas; 3) predominio de los núcleos, que contrasta con el bajo número de lascas; 4) el objetivo primordial de las secuencias de configuración, que afectó principalmente a las lascas, fue activar filos diédricos; 5) la diversidad de los tipos de configurados es sensiblemente superior en las lascas retocadas respecto a los artefactos sobre canto. Además, en el primero de estos soportes los instrumentos en que se asocian diversos diseños de 


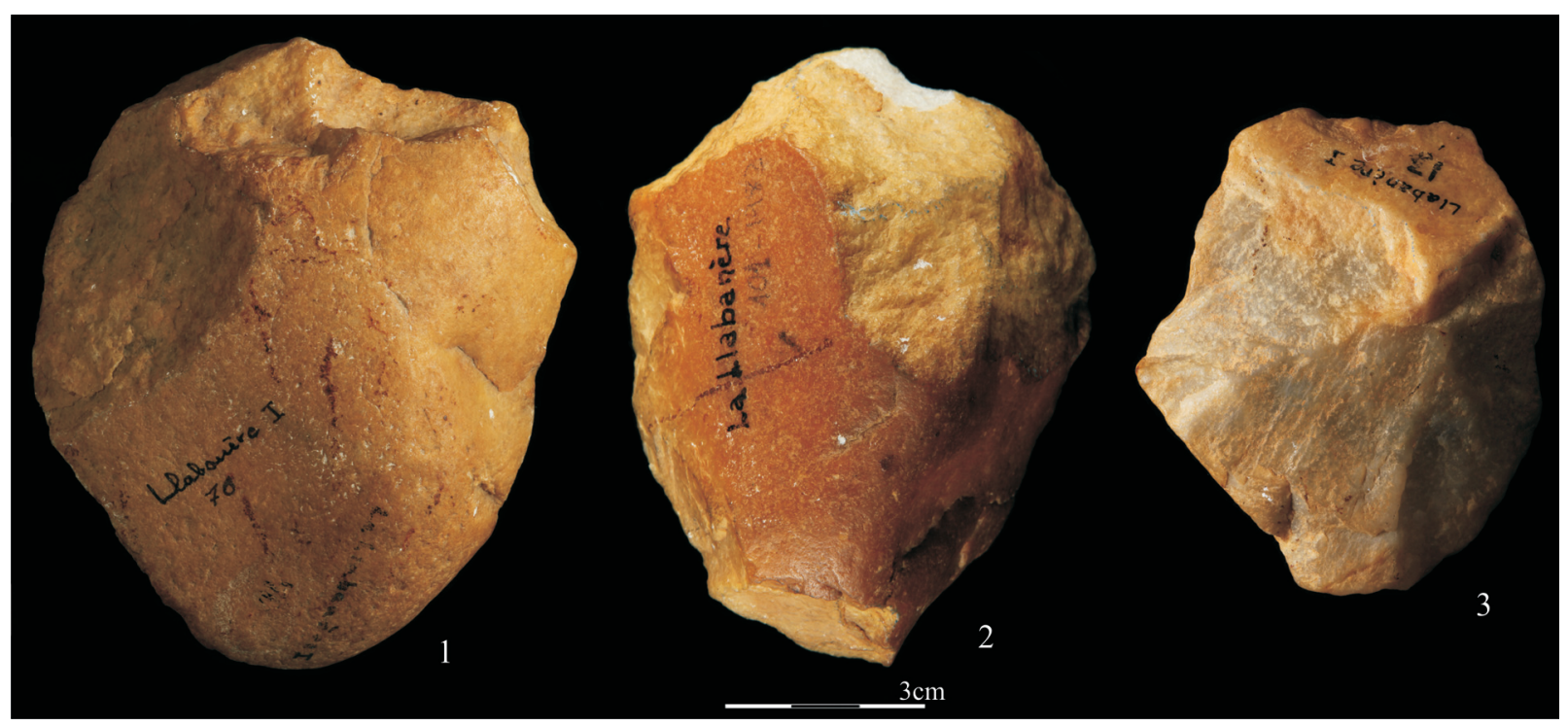

FIG. 9. Ejemplos de piezas de cuarzo con pátinas de eolización de la terraza de la Butte du Four-Llabanère (fotografias: de D. Dainat, EPCC-CERP, Tautavel).

configuración son más numerosos; 6) relativa especialización de los sistemas de configuración sobre canto -choppers y chopping-tools-; 7) destacada importancia de los bifaces; 8) diversidad técnica de los sistemas de explotación, que denota una gran intensidad de las secuencias de explotación; 9) destacan a nivel cuantitativo los métodos unifaciales y bifaciales unipolares de fácil elaboración, y a nivel cualitativo los sistemas bifaciales multipolares centrípetos -Levallois-; y 10) las cadenas operativas de configuración y explotación son completas y de amplio desarrollo técnico, documentándose tanto artefactos como núcleos sobre canto y lasca, así como sus correspondientes lascas (Figs. 11 y 13).

\section{Discusión}

\subsection{Continuum tecnológico}

Las industrias del sistema de terrazas del Rosellón permiten analizar la introducción en los sustratos tecnológicos regionales durante el Paleolítico inferior de ciertos cambios artefactuales, así como el mantenimiento de varias estructuras técnicas (Fig. 8). Todas presentan una importante homogeneidad tecnológica, especialmente detectable en
Mas Ferréol y la Plane d'en Bourgat. Este criterio, junto con el de las pátinas, con un nivel de erosión eólica similar entre las industrias de cada terraza, permiten descartar mezclas de materiales de épocas diferentes. Los análisis de los fenómenos de eolización de la industria lítica de la Butte du Four-Llabanère la sitúan como la más intensamente afectada por estos procesos (Fig. 9). La primera fase de eolización importante se ha podido constatar en el depósito y las industrias rissianas de esta terraza -que presentan además las mismas características sedimentológicas y pedológicas- y en los objetos líticos de la Plane d'en Bourgat. Posteriormente a la deposición de estas dos terrazas tuvo lugar una segunda fase de eolización de menor intensidad, que según Collina-Girard (1976, 1976c) se produjo durante la glaciación del Riss. Esta segunda fase parece tener su correlación directa con los niveles de limos de origen eólico de la vecina Caune del Arago (Tautavel), donde además se han recuperado algunos elementos líticos intensamente eolizados. En momentos avanzados del Würm tuvo lugar una última fase de eolización, que volvió a actuar sobre las industrias de la Butte du Four-Llabanère ${ }^{23}$.

${ }^{23}$ Collina-Girard: op. cit. n. 2. 


\begin{tabular}{|c|c|c|c|c|c|c|c|c|}
\hline \multicolumn{9}{|c|}{ Terraza de Mas Ferréol } \\
\hline & \multirow{3}{*}{$\begin{array}{c}\text { Percutores } \\
1\end{array}$} & \multicolumn{3}{|c|}{ Cantos } & Lascas & $\begin{array}{c}\text { Lascas } \\
\text { retocadas }\end{array}$ & \multirow{2}{*}{\multicolumn{2}{|c|}{ Total }} \\
\hline \multirow[b]{2}{*}{ Cuarzo } & & \multirow{2}{*}{$\frac{\text { Indet. }}{5}$} & \multirow{2}{*}{$\begin{array}{c}\text { Útiles } \\
20 \\
\end{array}$} & \multirow{2}{*}{$\begin{array}{c}\text { Núcleos } \\
60\end{array}$} & \multirow{2}{*}{6} & \multirow{2}{*}{33} & & \\
\hline & & & & & & & 125 & 98,4 \\
\hline Granito & - & - & 1 & - & - & - & 1 & 0,8 \\
\hline Arenisca & - & - & - & 1 & - & - & 1 & 0,8 \\
\hline \multirow{2}{*}{ Total } & 1 & 5 & 21 & 61 & 6 & 33 & \multirow{2}{*}{\multicolumn{2}{|c|}{127}} \\
\hline & 0,8 & 3,9 & 16,6 & 48,1 & 4,7 & 25,9 & & \\
\hline
\end{tabular}

\begin{tabular}{|c|c|c|c|c|c|c|c|c|c|}
\hline \multicolumn{10}{|c|}{ Terraza de la Plane d'en Bourgat } \\
\hline & \multirow{3}{*}{\begin{tabular}{|c|} 
Percutores \\
2 \\
\end{tabular}} & \multicolumn{3}{|c|}{ Cantos } & \multirow[t]{2}{*}{ Lascas } & \multicolumn{2}{|c|}{ Lascas } & \multirow{2}{*}{\multicolumn{2}{|c|}{ Total }} \\
\hline \multirow[b]{2}{*}{ Cuarzo } & & \multirow{2}{*}{$\frac{\text { Indet. }}{3}$} & \multirow{2}{*}{$\begin{array}{c}\text { Útiles } \\
12\end{array}$} & \multirow{2}{*}{\begin{tabular}{c|} 
Núcleos \\
49
\end{tabular}} & & \multirow{2}{*}{$\begin{array}{c}\text { Útiles } \\
19\end{array}$} & \multirow{2}{*}{\begin{tabular}{|c|} 
Núcleos \\
- \\
\end{tabular}} & & \\
\hline & & & & & 4 & & & 89 & 72,4 \\
\hline Cuarcita & - & 0 & 4 & 3 & 2 & 2 & - & 11 & 8,9 \\
\hline Arenisca & - & 1 & 2 & 1 & 1 & - & - & 5 & 4,1 \\
\hline Corneana & - & 1 & - & - & - & - & - & 1 & 0,8 \\
\hline Calcedonia & - & - & - & - & 1 & 4 & - & 5 & 4,1 \\
\hline Calcárea & - & 1 & 1 & - & - & - & 1 & 3 & 2,4 \\
\hline Granito & - & - & 2 & - & - & 1 & - & 3 & 2,4 \\
\hline Lidita & - & - & - & 1 & - & - & - & 1 & 0,8 \\
\hline Pórfido & - & - & - & 1 & - & 1 & - & 2 & 1,7 \\
\hline Sílex & - & - & - & 2 & 1 & - & - & 3 & 2,4 \\
\hline \multirow{2}{*}{ Total } & 2 & 6 & 21 & 57 & 9 & 27 & 1 & \multirow{2}{*}{\multicolumn{2}{|c|}{123}} \\
\hline & 1,6 & 4,9 & 17,1 & 46,4 & 7,3 & 21,9 & 0,8 & & \\
\hline
\end{tabular}

\begin{tabular}{|c|c|c|c|c|c|c|c|c|c|}
\hline \multicolumn{10}{|c|}{ Terraza de la Butte du Four-Llabanère } \\
\hline & Percutores & \multicolumn{3}{|c|}{ Cantos } & \multirow[t]{2}{*}{ Lascas } & \multicolumn{2}{|c|}{ Lascas } & \multirow{2}{*}{\multicolumn{2}{|c|}{ Total }} \\
\hline \multirow[b]{2}{*}{ Cuarzo } & \multirow[b]{2}{*}{7} & \multirow{2}{*}{$\begin{array}{c}\text { Indet. } \\
11\end{array}$} & \multirow{2}{*}{$\begin{array}{c}\text { Útiles } \\
42 \\
\end{array}$} & \multirow{2}{*}{$\begin{array}{c}\text { Núcleos } \\
470 \\
\end{array}$} & & \multirow{2}{*}{$\begin{array}{c}\text { Útiles } \\
152 \\
\end{array}$} & \multirow{2}{*}{\begin{tabular}{|c|} 
Núcleos \\
31 \\
\end{tabular}} & & \\
\hline & & & & & 164 & & & 877 & 98,3 \\
\hline Cuarcita & - & - & - & 3 & - & 2 & - & 5 & 0,6 \\
\hline Granito & - & 1 & 1 & 1 & - & - & - & 3 & 0,3 \\
\hline Jaspe & - & - & 1 & 3 & - & - & - & 4 & 0,5 \\
\hline Calcárea & - & - & 1 & - & - & - & - & 1 & 0,1 \\
\hline Lidita & - & - & - & 1 & - & - & - & 1 & 0,1 \\
\hline Calcedonia & - & - & - & 1 & - & - & - & 1 & 0,1 \\
\hline \multirow{2}{*}{ Total } & 7 & 12 & 45 & 479 & 164 & 154 & 31 & \multirow{2}{*}{\multicolumn{2}{|c|}{892}} \\
\hline & 0,8 & 1,3 & 5,0 & 53,7 & 8,4 & 7,3 & 3,5 & & \\
\hline
\end{tabular}

Fig. 10. Tipos de objetos y materias primas de la industria lítica de las terrazas de Mas Ferréol, Plane d'en Bourgat y Butte du Four-Llabanère.

En general, en las dos terrazas más antiguas se documenta una importante presencia de choppers -con escasos chopping-tools- entre los útiles sobre canto, con ausencia de bifaces, además de la introducción de las secuencias de explotación bifaciales centrípetas - discoidales-, encontrándose representado el sistema Levallois únicamente en la Plane d'en Bourgat. En las estrategias de explotación son importantes los núcleos multifaciales pertenecientes a sistemas de talla más expeditivos y de un menor nivel de organización técnica -poliedros-. La representación de estos elementos continúa siendo muy importante en la terraza de la Butte du Four-Llabanère, como consecuencia de una perduración tecnológica. Sin embargo, en las industrias de esta terraza destaca una mayor representación de las estrategias de explotación unifaciales y bifaciales centrípetas -discoides y Levallois-, con un mayor nivel de organización de la talla, apuntando a un incremento de la complejidad conceptual y operativa.

En el transcurso de la serie de terrazas, se observa una creciente estandarización y sistematización de los artefactos. En contraposición a la aparición de los primeros bifaces en la Butte du Four-Llabanère, perduran los choppers -configurados habitualmente a partir de planos de percusión corticales y otras veces de superficies generadas por una fractura-, que muestran una importante variación morfológica a lo largo de la secuencia de terrazas. De hecho, la variabilidad del perímetro trabajado y la delineación de los filos activados evidencia la ausencia de patrones sistemáticos en los procesos de configuración en las terrazas antiguas -Mas 
Ferréol y Plane d'en Bourgat-. Asimismo, las estrategias de configuración de artefactos y de obtención de lascas confluyen técnicamente en ciertos soportes. Es el caso de los chopping-tools con algunos núcleos bifaciales unipolares que muestran una misma ambivalencia técnica, así como una variabilidad tecnológica más acentuada que la de los choppers. En la misma línea están los bifaces -clasificados como abbevillienses por Collina-Girard- ${ }^{24}$ y ciertos núcleos bifaciales centrípetos, especialmente los de talla Levallois, presentan la misma confluencia operativa (Garcia Garriga, 2008, 2010, 2011).

También se detecta un importante incremento del número de chopping-tools en las terrazas más recientes, que aumentan su presencia en relación a los choppers. Asimismo, se registra la aparición de los primeros bifaces, todavía toscos y con un escaso nivel de configuración (Fig. 11). Igualmente, los núcleos bifaciales centrípetos -epannelés- y de estructura volumétrica bicónica -discoides, aunque en porcentajes no demasiado significativos- muestran también un aumento gradual en las terrazas más modernas. Las lascas continúan estando retocadas en porcentajes poco significativos, pero la ausencia de córtex en las caras dorsal y talonar y el mayor número de aristas las relacionan con una explotación bifacial técnicamente más compleja. Esta industria se asocia a un conjunto artefactual fuertemente dominado por los cantos tallados, produciéndose a la vez un ligero aumento de los núcleos Levallois. La escasa presencia de núcleos poliédricos o multifaciales -de un 4 a un $12 \%$ - en los yacimientos nos impide inferir una tendencia evolutiva diacrónica clara, si bien los porcentajes de estos objetos se presentan bastante repartidos en los diferentes conjuntos (Fig. 11). En líneas generales, en las series más recientes se aprecia un aumento paulatino y generalizado de la estandarización tipométrica y morfotécnica, tanto del conjunto de artefactos configurados como de los núcleos explotados y lascas producidas y retocadas. Pero, al mismo tiempo, perduran los choppers y chopping-tools, así como los núcleos gestionados con métodos multifaciales no sistemáticos - poliedros-, que muestran aristas sinuosas e irregulares.

24 Ibidem.
Según de Lumley et al. (1969a), los yacimientos de Mas Ferréol y le Correch contendrían las industrias de Modo 1 más antiguas, adscribibles al Donau -1.8 Ma-, mientras que las de la Llabanère y Manadeil serían del Modo 2 y posteriores en el tiempo, seguramente del Günz -1.1 Ma-. En cambio, para Collina-Girard (1976b, 1978) ${ }^{25}$ la periodización comenzaría con las industrias de Mata Roudoume -del Donau- y continuaría con las de Mas Ferréol -del Günz-, Cabestany -del Günz-Mindel; c. 750 ka-, Butte du Four -de inicios del Mindel; c. $650 \mathrm{ka}-$, Llabanère -de mediados del Mindel; c. $580 \mathrm{ka}-\mathrm{y}$ Pia -de finales de este periodo; c. $450 \mathrm{ka}$.

En un trabajo posterior de Lumley (1976) acabó por situar las industrias descubiertas en los enclaves de Mata Roudoume, Mas Ferréol y Cabestany en el Günz, manteniendo el esquema cronológico propuesto por Collina-Girard para el resto de terrazas. No obstante, los resultados anteriormente expuestos de los estudios sedimentológi$\cos ^{26}$ y geomorfológicos (Clauzon, 1984; Duvail et al., 2001; Giret, 1995) ${ }^{27}$ más recientes para estas formaciones cuaternarias convergen en un esquema evolutivo diferente, en el que nosotros nos hemos basado en este artículo. Así pues, sería más correcto atribuir a las industrias de Mas Ferréol, en las que también habría que incluir las de Mata Roudoume, al Günz, y de Cabestany (actualmente de la Plane d'en Bourgat) al Mindel. Para estos autores, la Butte du Four y la Llabanère formarían parte de la misma estructura fluvial, por lo que la hemos denominado Butte du Four-Llabanère, adscribible al Riss $-c .200 \mathrm{ka}-$, mientras que la de Pia correspondería, para Clauzon (1984) y Duvail et al. (2001), al Würm -c. 80 ka- (Fig. 5).

\subsection{Comparación tecnológica regional}

A un nivel regional más amplio, la industria de la cuenca del Rosellón puede relacionarse a nivel tecnológico con la de los yacimientos de la Caune de l'Arago -nivel G-, cuenca de los ríos Garona, Dordoña y Lot $-\mathrm{S}$ de Francia- y Ter y

${ }^{25}$ Cf. también op. cit. n. 2.

${ }^{26}$ Debals: op. cit. n. 3.

${ }^{27}$ Cf. también Calvet: op. cit. n. 3. 


\begin{tabular}{|l|c|c|c|}
\hline \multicolumn{3}{|c|}{ Sistemas de configuración } \\
\hline \multicolumn{3}{|c|}{ Cantos } \\
\hline \multirow{2}{*}{ Terrazas } & Diedro & Diedro+triedro & \multirow{2}{*}{ Total } \\
\cline { 2 - 3 } & choppers/ & bifaces & \\
\cline { 2 - 3 } & chopping-tools & & 21 \\
\hline Terraza de Mas Ferréol & 21 & - & 21 \\
\hline Terraza de la Plane d'en Bourgat & 21 & - & 46 \\
\hline Terraza de la Butte du Four-Llabanère & 43 & 3 & 88 \\
\hline \multirow{2}{*}{ Total } & 85 & 3 & \\
\cline { 2 - 4 } & 96,6 & 3,4 & \\
\hline
\end{tabular}

\begin{tabular}{|c|c|c|c|c|c|}
\hline \multicolumn{6}{|c|}{ Lascas } \\
\hline \multirow{3}{*}{ Terrazas } & Diedro & Ddro dent cc & Triedro & $\begin{array}{c}\text { Diedro + } \\
\text { triedro }\end{array}$ & Total \\
\hline & \multirow{2}{*}{ raederas } & denticulados/ & \multirow{2}{*}{ puntas } & \multirow{2}{*}{ bifaces } & \\
\hline & & muescas & & & \\
\hline Terraza de Mas Ferréol & 8 & 25 & - & - & 33 \\
\hline Terraza de la Plane d'en Bourgat & 12 & 14 & 1 & - & 27 \\
\hline Terraza de la Butte du Four-Llabanère & 65 & 88 & - & 1 & 154 \\
\hline \multirow{2}{*}{ Total } & 85 & 127 & 1 & 1 & \multirow{2}{*}{214} \\
\hline & 39,7 & 59,3 & 0,5 & 0,5 & \\
\hline
\end{tabular}

\begin{tabular}{|l|c|c|c|c|c|}
\hline \multicolumn{7}{|c|}{ Sistemas de explotación } \\
\hline \multirow{2}{*}{ Terrazas } & $\begin{array}{c}\text { U/B } \\
\text { unipolar }\end{array}$ & $\begin{array}{c}\text { U/B } \\
\text { bipolar } \\
\text { opuesto }\end{array}$ & $\begin{array}{c}\text { U/B } \\
\text { bipolar } \\
\text { ortogonal }\end{array}$ & $\begin{array}{c}\text { U/B } \\
\text { multipolar } \\
\text { ortogonal }\end{array}$ & $\begin{array}{c}\text { U/B } \\
\text { unipolar/ } \\
\text { bipolar } \\
\text { circular }\end{array}$ \\
\hline Terraza de Mas Ferréol & 35 & 5 & 3 & 2 & - \\
\hline Terraza de la Plane d'en Bourgat & 24 & 2 & 8 & 6 & 1 \\
\hline Terraza de la Butte du Four-Llabanère & 198 & 45 & 73 & 63 & 20 \\
\hline \multirow{2}{*}{ Total } & 257 & 52 & 84 & 71 & 21 \\
\cline { 2 - 6 } & 39,4 & 7,9 & 12,9 & 10,9 & 3,2 \\
\hline
\end{tabular}

\begin{tabular}{|c|c|c|c|c|c|}
\hline \multirow[t]{3}{*}{ Terrazas } & \begin{tabular}{c|}
$\mathrm{U} / \mathrm{B}$ \\
multipolar \\
centrípeto \\
\end{tabular} & \begin{tabular}{|c|}
$\mathrm{B}$ \\
multipolar \\
centrípeto \\
\end{tabular} & \multirow[t]{3}{*}{ T multipolar } & M multipolar & \multirow[t]{3}{*}{ Total } \\
\hline & epannelés/ & \multirow{2}{*}{ Levallois } & & \multirow{2}{*}{ poliedros } & \\
\hline & discoides & & & & \\
\hline Terraza de Mas Ferréol & 2 & - & 6 & 6 & 59 \\
\hline Terraza de la Plane d'en Bourgat & 8 & 2 & 5 & 2 & 58 \\
\hline Terraza de la Butte du Four-Llabanère & 50 & 6 & 67 & 14 & 536 \\
\hline \multirow{2}{*}{ Total } & 60 & 8 & 78 & 22 & \multirow{2}{*}{653} \\
\hline & 9,2 & 1,2 & 11,9 & 3,4 & \\
\hline
\end{tabular}

FIG. 11. Estrategias de configuración de artefactos y de explotación desarrolladas en las terrazas de Mas Ferréol, Plane d'en Bourgat y Butte du Four-Llabanère (Ddro dent cc $=$ Diedro denticulado cóncavo; $U=$ Unifacial; $B=$ Bifacial; $T=$ Trifacial y $M=$ Multifacial). depresión de la Selva -NE de Cataluña-, asociados mayoritariamente al uso especializado del cuarzo (Bruxelles et al., 2003; Turq, 2000; Garcia Garriga, 2008, 2010, 2011) ${ }^{28}$. La tendencia general en estas zonas está marcada por el aprovisionamiento de las materias primas necesarias para la talla dentro de un radio de acción inferior a $5 \mathrm{~km}$ del lugar de ocupación. El estudio comparativo de la gestión de las materias primas ha permitido detectar un patrón común en la mayoría de los yacimientos: su utilización diferenciada y la tendencia hacia la especialización técnica de determinadas materias como el cuarzo, la cuarcita o el sílex, según la disponibilidad de los tipos de roca respecto al lugar de ocupación.

En las secuencias de configuración de las industrias de esta región, tanto sobre canto como sobre lasca, la tendencia fue la de confeccionar útiles mediante sistemas unifaciales -choppers en los útiles sobre canto y raederas, denticulados y muescas en las lascas retocadas-. Sin embargo, en la Plane d'en Bourgat y la Butte du Four-Llabanère predominan los segmentos de las lascas con retoque bifacial. En cuanto a los tipos de útiles sobre canto se observa una cierta sistematización de los segmentos diédricos-choppers y chopping-tools, con muy escasos hendedores-, mientras que los triedros - pi$\cos$ y bifaces- aparecen en los conjuntos líticos siempre de manera muy puntual. Los

${ }^{28}$ Cf. también Turq: op. cit. n. 4. 
útiles retocados también se componen de diedros continuos -raederas-, denticulados y cóncavos -muescas-, que presentan proporciones variables según los yacimientos. Así, los bordes diédricos sobre lasca con retoques denticulados y cóncavos predominan en muchos yacimientos -terraza de la Butte du Four-Llabanère, Puig d'en Roca Excavació, nivel 1 de Can Garriga y los tecnocomplejos de la Selva-, especialmente en la terraza de Mas Ferréol y en otros conjuntos de la cuenca del Ter -Puig d'en Roca III y IV y Costa Roja-. Las lascas con segmentos continuos -raederas- son más comunes en el nivel G de la Caune de l'Arago y en ciertos yacimientos de las cuencas del Ter (Puig d'en Roca I-II, nivel 2 Can Garriga y Cau del Duc de Torroella de Montgrí), Lot -Coudoulous, Carrière de Billon, Bourg-de-Tombeboeuf y Les Combes- y Dordoña -Roquepine, Combe-Grenal, Grotte Vauffrey y La Plane-. Las industrias de la terraza de la Plane d'en Bourgat y de otros yacimientos de las terrazas del Lot -Tuc-de-Bardety Ter -Costa Roja, Mas d'en Galí, Domeny Industrial y Cau del Duc d'Ullà- muestran un equilibrio entre los dos modelos.

Los sistemas de explotación permiten observar la existencia
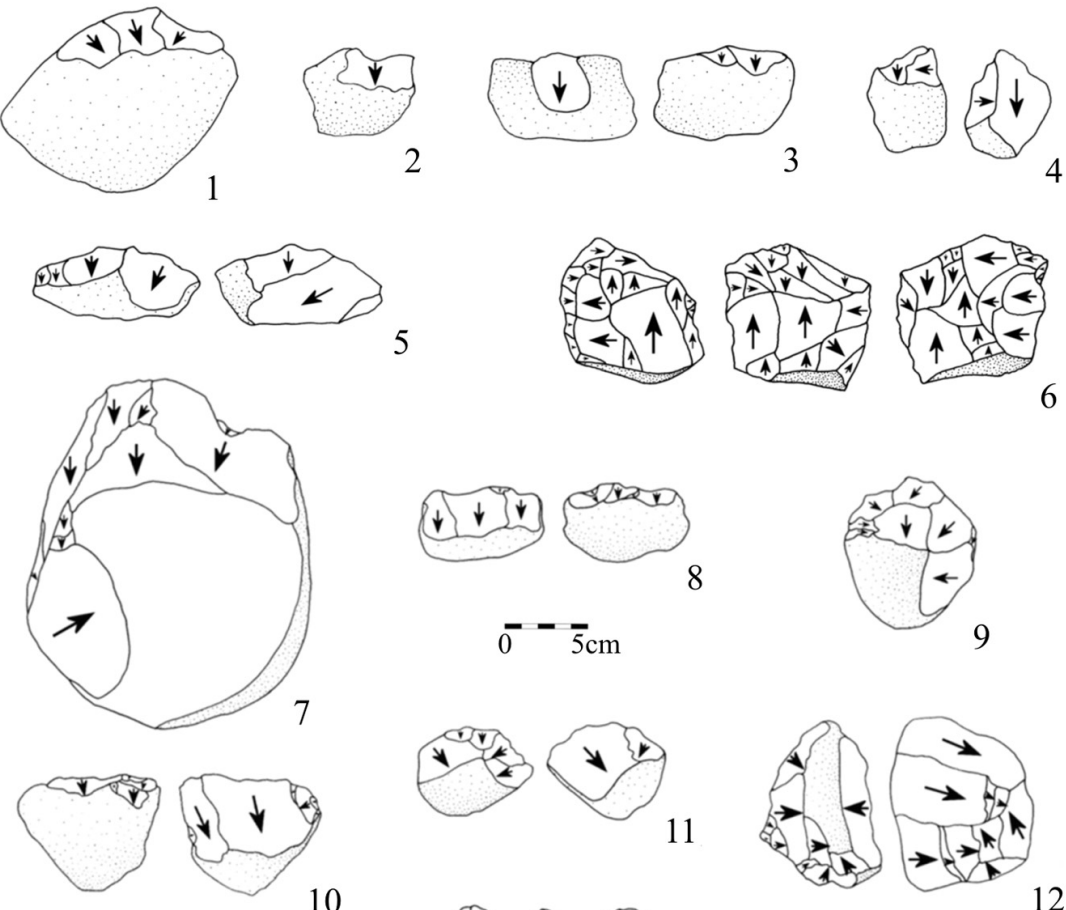

$\overline{0}-\overline{5} \mathrm{~cm}$

9
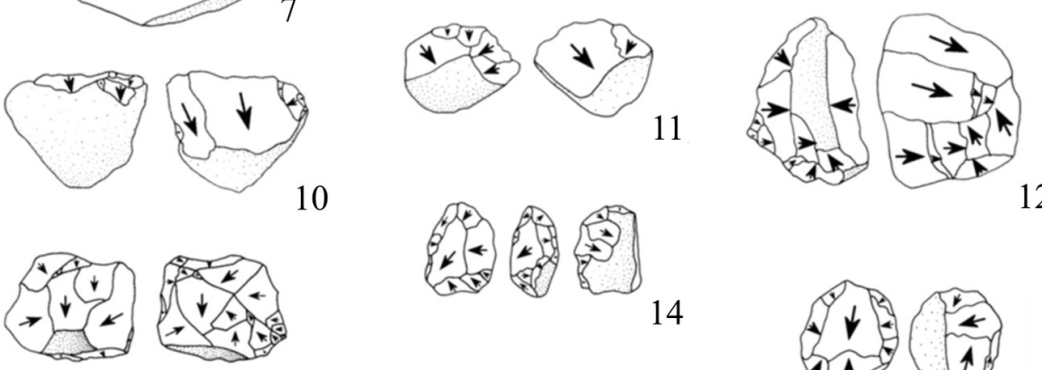

10

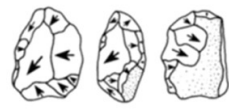

14

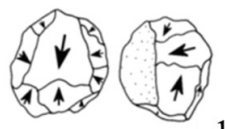

13
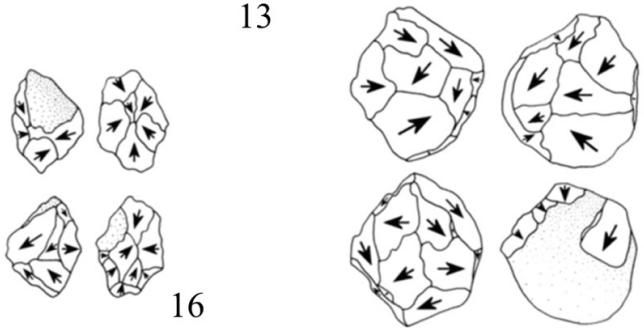

15

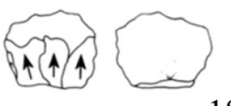

18

\section{6}

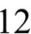

Fig. 12. Dibujos técnicos de la industria lítica de Mas Ferréol (n.os 1-6): 1) chopper; 2-5) núcleos unifaciales y bifaciales unipolares y bipolares y 6) núcleo trifacial. Industria de la Plane d'en Bourgat (n. ${ }^{o s}$ 7-18): 7) chopper; 811) núcleos unifaciales y bifaciales unipolares y bipolares; 12-13) núcleos bifaciales ortogonales; 14-15) núcleos bifaciales centripetos; 16-17) núcleos trifaciales y multifaciales y 18) lasca unipolar.

de una importante diversidad

tecnológica en todas las regiones próximas al Rosellón. Entre los yacimientos que presentan una mayor diversidad de sistemas se encuentran las terrazas de la Plane d'en Bourgat y Butte du Four-Llabanère, el nivel G de la Caune de l'Arago y algunos yacimientos del Ter -Costa Roja, Puig d'en Roca Excavació y III, niveles 1 y 2 de Can Garriga y Cau del Duc de Torroella de Montgrí-, Lot -Les Combes-, Dordoña -La Plane Mare, Combe-Grenal y Grotte Vauffrey- y la Selva. Por otra parte, en la terraza de Mas Ferréol, en yacimientos del Lot -en Tuc-de-Bardet, Carrière de Billon y Bourgde-Tombeboeuf, además de un único núcleo poliédrico en Coudoulous-, Dordoña-Roquepine 

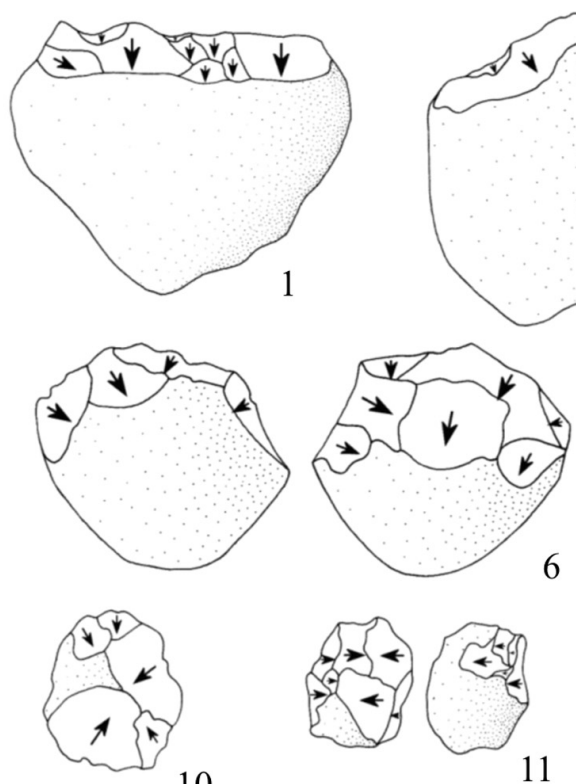

10

11

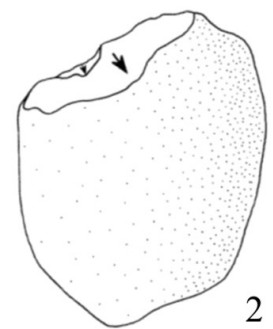

2

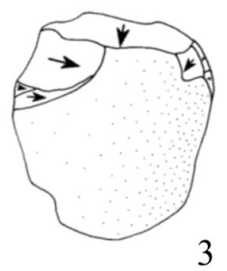

3
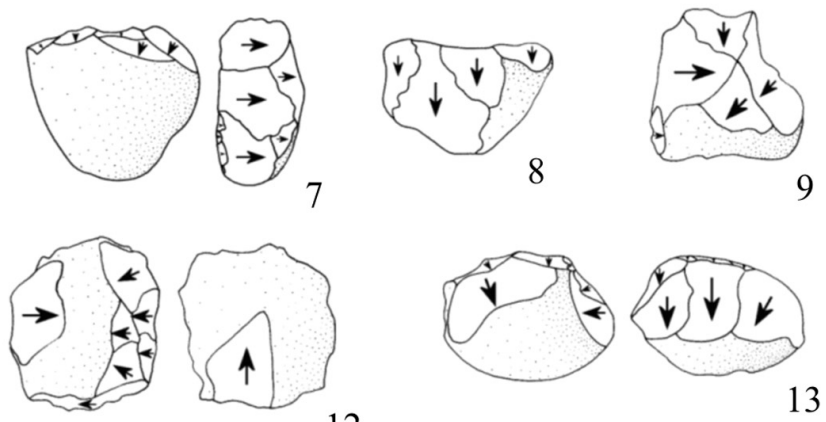
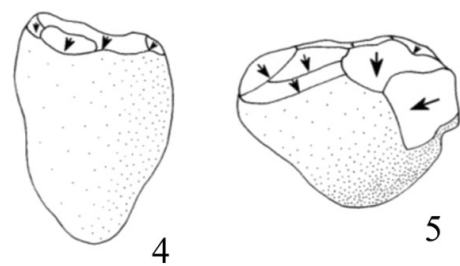

5

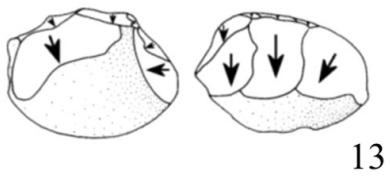

12
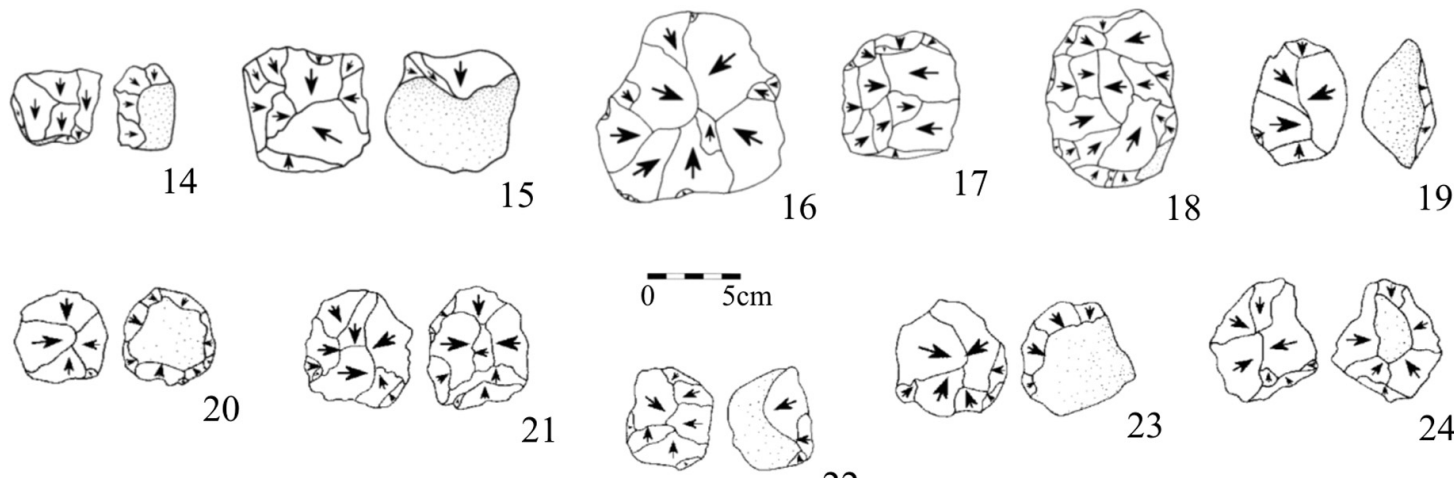

22
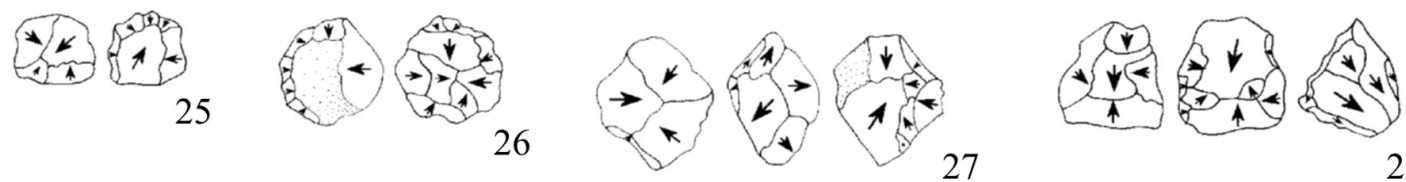

27

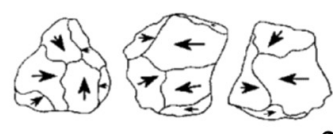

29
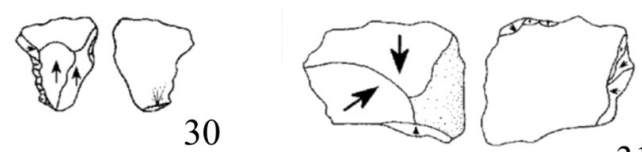

31

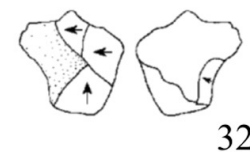

32
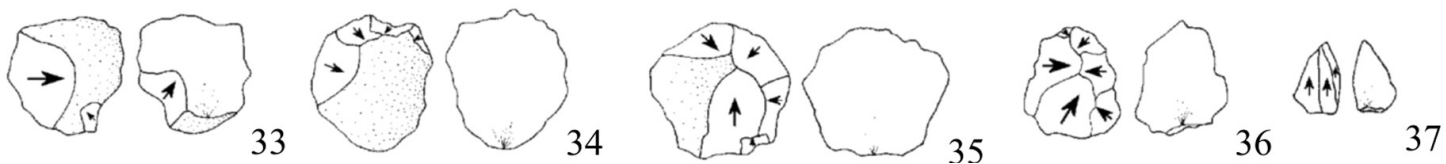

FIG. 13. Dibujos técnicos de la industria lítica de la Butte du Four-Llabanère: 1-5) choppers; 6) chopping-tool; 7-8 y 11-14) núcleos unifaciales y bifaciales unipolares y bipolares; 9, 10 y 15) núcleos unifaciales y bifaciales ortogonales; 16-27) núcleos unifaciales y bifaciales centripetos; 28-29) núcleos trifaciales; 30-31) raederas; 32-33) muescas; 34 y 37) lascas unipolares y 35 y 36) lascas centripetas. 
y Grotte Vauffrey-, Garona -Rominguière- y en otros conjuntos del Ter-Mas d'en Galí y Domeny Industrial-, la especialización técnica de ciertos sistemas de reducción fue más importante. En las cuencas del Rosellón y el Ter, donde el cuarzo es la roca más abundante, y también del Lot, Dordoña y Garona, aparecen sistemas que presentan un cierto nivel de complejidad como el discoide y Levallois, lo que nos permite afirmar que la disponibilidad de materias primas no condicionó en general los sistemas de explotación.

Tanto en la talla de núcleos sobre canto como sobre lasca, se documenta el uso generalizado de la talla bifacial. En los yacimientos de los territorios septentrionales de Cataluña y del s francés, aunque las gestiones bifaciales son generalmente mayoritarias en las actividades de producción, también se constata una presencia significativa de la talla trifacial, multifacial -con algunos poliedros- y sobre todo unifacial -con algunos epannelés-. Los núcleos bifaciales predominan en el nivel G de la Caune de l'Arago, Ter -Puig d'en Roca Excavació, Domeny Industrial, niveles 1 y 2 de Can Garriga y Cau del Duc de Torroella de Montgrí-, Dordoña -La Plane Mare, Roquepine y Grotte Vauffrey-, Garona -Rominguière- y la Selva, mientras que las estrategias unifaciales son más comunes en las terrazas del Rosellón y en otros yacimientos del Ter -Costa Roja y Mas d'en Galí- y Dordońa -Combe-Grenal-.

\subsection{Modelos de aprovisionamiento de materias primas y tradiciones técnicas}

Según el radio de aprovisionamiento de las materias primas, tanto en la cuenca del Rosellón como en el resto de yacimientos coetáneos del NE peninsular y $\mathrm{S}$ francés se documenta un modelo de captación local (Bruxelles et al., 2003; Turq, 2000; Garcia Garriga, 2008, 2010, 2011) ${ }^{29}$. Este análisis territorial aporta aspectos inferenciales en cuanto a las estrategias de movilidad espacial y de explotación del territorio a gran escala. Las actividades desarrolladas por los homínidos para la obtención de las rocas necesarias para desarrollar las actividades técnicas están ligadas estrictamente

${ }^{29}$ Cf. también op. cit. n. 4. a su comportamiento paleoeconómico, a la extensión territorial y a las áreas frecuentadas (Geneste, 1991). En todos los yacimientos del territorio en cuestión se documenta un comportamiento de movilidad relacionado con la instalación recurrente de las ocupaciones en puntos estratégicos cercanos y de fácil acceso a las áreas de captación de materias primas.

Para la Caune de l'Arago (Tautavel), sin embargo, el aprovisionamiento puntual de ciertos materiales como el sílex o el jaspe tuvo lugar en un radio de acción más amplio, llegando a superar áreas superiores a $30 / 40 \mathrm{~km}$ respecto a la cueva (Grégoire, 2000; de Lumley, 2000; de Lumley et al., 2004). Pese a que hemos asociado estas rocas a chaînes opératoires fragmentadas, esta interpretación supone la existencia de un comportamiento tecnoeconómico ocasional respecto al resto de territorios próximos. Este modelo se ha podido documentar gracias a la alta resolución arqueológica que presenta el depósito de la Caune de l'Arago, lo cual no sucede en los yacimientos en superficie estudiados. Fernández-Peris y Villaverde (1996) documentan la existencia de objetos líticos procedentes de zonas más allá de los 60 $\mathrm{km}$, que corresponderían, según Geneste (1991), a áreas escasamente frecuentadas. Para los yacimientos del $\mathrm{s}$ francés, se podría demostrar la existencia de radios de acción más cercanos y frecuentados de unos $20 \mathrm{~km}$ en relación a los asentamientos. Pero, incluso en este caso, se continúa manteniendo como modelo general unos patrones de movilidad inferiores a los $5 \mathrm{~km}$ de los lugares de ocupación. Este hecho estaría relacionado con el principio de rentabilidad energética, según la cual se llevaría a cabo una explotación frecuente de los recursos naturales más próximos y abundantes en función de la relación coste-economía energética -según los modelos teóricos elaborados por Bettinger y Baumhoff (1982), Binford (1980), Dyson-Hudson y Smith (1978), Krebs y Davies (1984), Orians y Pearson (1979) y Querol (1985)-.

Para el NE peninsular y $\mathrm{S}$ francés, desde mediados del Pleistoceno medio hasta inicios del Pleistoceno superior, se ha propuesto la presencia de estructuras tecnológicas singulares en cada región, que mostrarían un margen de variabilidad entre las distintas unidades ecológicas. En efecto, se 
observa una homogeneidad tecnológica entre los sistemas de producción técnicos que forman parte de una misma unidad territorial. Las mínimas variaciones tecnológicas intrarregionales serían el producto de adaptaciones funcionales específicas adoptadas por unas mismas comunidades regionales, como consecuencia de posibles necesidades diferenciales, o debido a aspectos relacionados con la frecuencia, intensidad o duración de las ocupaciones. Esta hipótesis conllevaría el planteamiento de la presencia de poblaciones dotadas de sustratos culturales diferenciales, que pudieron perdurar en estas unidades espaciales tecnológicamente singularizadas (Garcia Garriga, 2008, 2010, 2011). La selección sistemática de pequeńos clastos y cantos para las secuencias de explotación del yacimiento del Pleistoceno inferior de Vallparadís (Terrassa), puesto que existían en las inmediaciones cantos de formatos mayores, también se ha interpretado como parte de unas tradiciones técnicas ligadas a la talla recurrente mediante el método bipolar sobre yunque de soportes de pequeño tamaño (Garcia Garriga et al., 2013). En ese trabajo, se propone que en el Modo 1 europeo habrían existido diversas tradiciones técnicas que explicarían la variabilidad de sus industrias.

Desde este mismo punto de vista culturalista, Turq $^{30}$ interpreta las heterogeneidades tecnológicas de las industrias del Paleolítico inferior y medio de los valles de Dordońa y Lot en función de la existencia de "facies culturales" diferenciadas. Estas diferentes tradiciones técnicas estarían motivadas por las adaptaciones tecnológicas de los homínidos a los diversos entornos ecosistémicos ocupados. En el mismo sentido, Moncel (2003a) interpreta las industrias microlíticas de inicios del Pleistoceno superior de la Europa central -Tata, Külna y Predmosti IIcomo una misma unidad cultural que tendría sus orígenes en las industrias de Vértesszőlős, en Hungría. Esta misma autora interpreta las particularidades tecnológicas de las industrias del valle medio del Ródano según su filiación tecnológica o facies cultural, definida por la recurrencia de la talla Levallois, discoide, laminar o Quina y la ausencia de útiles retocados (Moncel,

${ }^{30}$ Cf. también op. cit. n. 4. 2003b). Estas diversas tradiciones técnicas habrían perdurado en esta región durante el MIS 9 y 3 (347-64 ka).

\section{Conclusiones}

La evolución de las industrias del Paleolítico inferior del valle del Têt está marcada en el registro litotécnico de las terrazas de Mas Ferréol y la Plane d'en Bourgat por un elevado número de cantos configurados poco estandarizados y de talla irregular, que predominan en su variedad diédrica unifacial -chopper-. Los núcleos y lascas, normalmente corticales en la terraza de Mas Ferréol, exhiben unos esquemas operativos elaborados mediantes chaînes opératoires escasamente desarrolladas propias del Modo 1, en los que raramente están incluidas las secuencias de configuración de las lascas. Éstas aumentan en la Plane d'en Bourgat, que corresponderían al Modo 2 -aunque sin bifaces y con muy escaso peso de las estrategias más estandarizadas como las Levallois-. Estas industrias podrían relacionarse con la primera aparición del Modo 2 en la región, fechada en unos $650 \mathrm{ka}$ en la cercana Caune de l'Arago (Barsky y de Lumley, 2010). En la terraza de la Butte du Four-Llabanère, se establecieron nuevas ocupaciones con una tecnología de Modo 2 con un utillaje más evolucionado, que ya disponía de bifaces, además de una mayor presencia de chopping-tools y de estrategias de gestión más elaboradas y sistematizadas -Levallois-. La estasis tecnológica de estas industrias, con la pervivencia en las industrias de Modo 2 de estructuras técnicas propias del Modo 1, principalmente cantos configurados, se mantuvo al menos desde finales del Pleistoceno inferior hasta el Pleistoceno medio tardío.

A escala regional, estas industrias comparten un mismo sustrato tecnológico con los yacimientos coetáneos de la Caune de l'Arago -nivel G-, cuenca de los ríos Garona, Dordoña y Lot $-\mathrm{S}$ de Francia- y Ter y depresión de la Selva -NE de Cataluña- (Turq, 2000; Bruxelles et al., 2003; Garcia Garriga, 2008, 2010, 2011) ${ }^{31}$. Estas industrias están definidas por el uso recurrente del

${ }^{31}$ Cf. también op. cit. n. 3. 
cuarzo y por su aprovisionamiento en radios de acción generalmente cortos, inferiores a los $5 \mathrm{~km}$ respecto a los lugares de ocupación. Por lo tanto, se puede definir un patrón de movilidad general ligada a la instalación de los grupos humanos en puntos estratégicos cercanos y de fácil acceso a las zonas de captación de materias primas. Las secuencias de configuración sobre canto están dirigidas a la obtención de útiles unifaciales -generalmente choppers y en menor medida chopping-tools-. Otros morfotipos como los picos, hendedores y bifaces aparecen ocasionalmente en algunos yacimientos. A diferencia de la Plane d'en Bourgat y la Butte du Four-Llabanère, donde los retoques de las lascas son bifaciales, en los yacimientos del NE peninsular y otros conjuntos del $\mathrm{S}$ francés suelen mostrar intervenciones unifaciales. Los artefactos retocados más habituales son las raederas, seguidas de denticulados y muescas. Mientras que los denticulados son más habituales en la Butte du Four-Llabanère, en la Plane d'en Bourgat existe un equilibrio entre denticulados y raederas. A diferencia de lo que sucede en Mas Ferréol, donde se documenta una especialización técnica en las cadenas de explotación, en la Plane d'en Bourgat y Butte du Four-Llabanère y en los territorios próximos al Rosellón son más diversificadas. La talla bifacial resulta predominante en este contexto regional, aunque en la cuenca del Rosellón la talla unifacial es muy significativa.

A pesar de estas similitudes tecnológicas en el NE peninsular y S francés, existe cierta variabilidad tecnológica entre los yacimientos que corresponden a las distintas unidades regionales (García Garriga, 2008, 2010, 2011). Este margen de variabilidad y las mínimas diferencias intrarregionales observadas permiten plantear la hipótesis de la existencia de poblaciones humanas provistas de sistemas tecnológicos singulares en las distintas regiones en las que habrían tenido lugar variaciones tecnológicas durante el Paleolítico inferior.

\section{Bibliografía}

AMBERT, R. (1993): L'évolution quaternaire du Languedoc central et des Corbières littorales déduite de séquences bio et chronostratigraphiquement bien calées. Livret-guide de l'excursion de L'AFEQ, XXII. URA 903. Paris: CNRS.
BARRIÈRE, J. (1969): "Les terrasses quaternaires de la plaine du Roussillon (Pyrénées-Orientales)”. En TAILlefer, F. (ed.): VIII Congrès de l'INQUA. Livretguide de l'excursion A6. Paris, pp. 73-78.

BARRIÈRe, J. y MichauX, J. (1973): "Essai de corrélation du plio-pléistocène languadocien". En $I X^{e}$ Congrès de l'INQUA. Christchurch.

Barsky, D. y De Lumley, H. (2010): "Early European Mode 2 and the stone industry from the Caune de l'Arago's archeostratigraphical levels 'P'", Quaternary International, 223, pp. 71-86. http://dx.doi.org/10.1016/j.quaint.2009.12.005

Bettinger, R. L. y Baumhoff, M. A. (1982): "The Numic Spread: Great Basin Cultures in Competition", American Antiquity, 47, pp. 485-503. http://dx.doi.org/10.2307/280231

BINFORD, L. R. (1980): "Willow Smoke and Dogs'tails: Hunter-gatherer Settlement Systems and Archaeological Site Formation", American Antiquity, 45, pp. 4-20. http://dx.doi.org/10.2307/279653

BOURCART, J. (1945): "Étude des sédiments pliocènes et quaternaires de la plaine du Roussillon", Bulletin du Service de la Carte Géologique de France, XLV (218), pp. 1-82.

Bruxelles, L.; Berthet, A.-L.; Chalard, P.; Colonge, D.; Delfour, G.; Jarry, M.; Lelouvier, L.-A.; Arnoux, T. y OnÉzime, O. (2003): "Le paléolithique inférieur et moyen en Midi toulousain: nouvelles données et perspectives de l'archéologie préventive", Paleo, 15, pp. 7-28.

Carbonell, E.; Cebrià, A.; Esteban, A.; Mora, R. y PARRA, I. (1983): "Aproximació crítica a l'estudi dels espais prehistòrics", Estudis d'Història Agrària, pp. 87-99.

Carbonell, E.; Rodríguez, X. P.; Sala, R. y VAQUERO, M. (1992): "New Elements of the Logical-analitic System", Cahier Noir, 6, pp. 3-61.

Clauzon, G. (1984): "Le Quaternaire du Roussillon". En Synthèse géologique du Sud-Est de la France. Mémoire du BRMG, 125, pp. 558-559.

Collina-Girard, J. (1976a): "Terrasses du Quaternaire ancien de la Têt". En De Lumley, H. (ed.): Provence et Languedoc méditterranéen. Sites paléolithiques et néolithiques. IX Congrès de l'UISSP. Livretguide de l'excursion C2. Nice, pp. 317-320.

COLlina-Girard， J. (1976b): "Les industries archaiques sur galet de la plaine du Roussillon". En De Lumley, H. (ed.): Provence et Languedoc méditterranéen. Sites paléolithiques et néolithiques. IXe Congrès de l'UISSP. Livret-guide de l'excursion C2. Nice, pp. 320-325.

COLLINA-GIRARD, J. (1976c): "Les alluvions fluviatiles des fleuves côtiers dans le Roussillon (Têt, Tech, 
Agly)”. En De Lumley, H. y Guilaine, J. (eds.): La Préhistoire Française, vol. I. Paris: Éditions du CNRS, pp. 78-82.

Collina-Girard, J. (1978): "Évolution des industries à galets aménagés de la vallée de la Têt (PyrénéesOrientales)", Bulletin de la Société Préhistorique Française, 75 (6), pp. 172-180. http://dx.doi.org/10.3406/bspf.1978.8577

De la Torre, I. y Mora, R. (2009): "Remarks on the current theoretical and methodological approaches to the study of early technological strategies in Eastern Africa”. En Hovers, E. y Braun, D. R. (eds.): Interdisciplinary approaches to the Oldowan. Dordrecht: Springer, pp. 15-24.

De Lumley, H. (1969a): "Les civilisations préhistoriques en France. Corrélations avec la chronologie quaternaire". En De Lumley, H. y Guilaine, J. (eds.): La Préhistoire Française, vol. I. Paris: Éditions du CNRS, pp. 151-169.

De Lumley, H. (1969b): "Les industries préhistoriques de la vallée de la Têt". En TAILlefer, F. (ed.): VIII Congrès de l'INQUA. Livret-guide de l'excursion A6. Paris, pp. 79-84.

De Lumley, H. (1971): Le Paléolithique inférieur et moyen du midi méditerranéen dans son cadre géologique. Ve supplément à Gallia Préhistoire, vols. I-II. Paris: Éditions du CNRS.

De Lumley, H. (1976): "Les civilisations du Paléolithique inférieur en Languedoc méditerranéen et en Roussillon”. En De Lumley, H. y Guilaine, J. (eds.): La Préhistoire Française, vol. I. Paris: Éditions du CNRS, pp. 852-874.

De Lumley, H. (2000): El primer hombre. Madrid: edit. Cátedra.

De Lumley, H.; Collina-Girard, J.; Abelanet, J.; Bazile, F. y Meignen, L. (1976): "Les premières industries humaines en Languedoc méditerranéen et en Roussillon". En De LuMLeY, H. y Guilaine, J. (eds.): La Préhistoire Française, vol. I. Paris: Éditions du CNRS, pp. 777-794.

De Lumley, H.; Grégoire, S.; Barsky, D.; Batalla, G.; Bailón, S.; Belda, V.; Briki, D.; Byrne, L.; Desclaux, E.; El Guenouni, K.; Fournier, A.; Kacimi, S.; Lacombat, F.; De Lumley, A. A.; Moigne, A. M.; Moutoussamy, J.; Paunescu, C.; Perrenoud, Ch.; Pois, V.; Quiles, J.; Rivals, F.; Roger, T. y Testi, A. (2004): "Habitat et mode de vie des chasseurs paléolithiques de la Caune de l'Arago (600000400000 ans)", L'Anthropologie, 108, pp. 159-184. http://dx.doi.org/10.1016/j.anthro.2004.05.001

Duvail, P.; Le Strat, G. y Clauzon, G. (2001): Synthèse cartographique d̀ $1 / 100.000$ des formations cénozoïques $d u$ bassin $d u$ Roussillon. Escala 1:100.000. Service du CDG/DPN/NDC.

Dyson-Hudson, R. y Smith, E. A. (1978): "Human Territoriality: an Ecological Reassessment", American Anthropologist, 80, pp. 21-41.

http://dx.doi.org/10.1525/aa.1978.80.1.02a00020

FernÁNDEZ-Peris, J. y VillaVerde, V. (1996): “The Role and Appraisal of Quartzite and Other Non-flint Lithic Materials in the Lower and Middle Palaeolithic of Valencia”. En MoloneY, N.; RAPOSO, L. y SAntonja, M. (eds.): Non-flint Stone Tools and the Palaeolithic Occupation of the Iberian Peninsula. BAR Int. Ser., 649. Oxford: Archaeopress, pp. 125-130.

Garcia Garriga, J. (2008): El Paleolitic inferior a Catalunya $i$ al Rosselló. Ripoll: Institut d'Estudis Ceretans.

Garcia Garriga, J. (2010): Tecnología lítica del Paleolítico inferior del noreste de la Peninsula Ibérica y sureste de Francia. BAR Int. Ser., 2101. Oxford: Archaeopress.

Garcia Garriga, J. (2011): "Continuité technologique et traditions techniques au Paléolithique inférieur : un modèle d'occupation territoriale dans le Sud de la France et dans le Nord-Est de la péninsule Ibérique", Bulletin de la Société Préhistorique Française, 108 (4), pp. 609-643.

García Garriga, J.; Martínez, K. y Carbonell, E. (2013): "The Early Pleistocene stone tools from Vallparadís (Barcelona, Spain): Rethinking the European Mode 1", Quaternary International, 316, pp. 94-114. http://dx.doi.org/10.1016/j.quaint.2013.09.038

Geneste, J.-M. (1991): "L'aprovisionnement en matières premières dans les systèmes de production lithique: la dimension spatiale de la technologie". En MorA, R. (ed.): Tecnología y cadenas operativas liticas. Barcelona: UAB, pp. 1-35.

Giret, A. (1995): "Étude des deformations quaternaires de la Têt par l'analyse morphométrique", Quaternaire, 6 (3-4), pp. 121-137. http://dx.doi.org/10.3406/quate.1995.2045

Got, H. (1967): "Contribution à l'étude sédimentologique des terrasses quaternaires du Roussillon", Bulletin de la Société Préhistorique Française, IX (7), pp. 212-220.

GUILBAUD, M. (1995): "Introduction sommaire au concept de champ opératoire", Cahier Noir, 7, pp. 121-133.

Hiscock, P. (2007): "Looking The Other Way: A Materialist/Technological Approach to Classifying Tools and Implements, Cores and Retouched Flakes". En McPherron, S.-P. (ed.): Tools versus Cores: Alternative Approaches to Stone Tool Analysis. 
Newcastle: Cambridge Scholars Publishing, pp. 198-222.

Krebs, J. R. y Davies, N. B. (1984; $2 .^{\text {a }}$ ed.): Behavioural Ecology. An Evolutionary Approach. Oxford: Blackwell Scientific Publications.

Kunn, S. L. (2007): "Cores, Tools, and the Priorities of Lithic Analysis". En McPherron, S.-P. (ed.): Tools versus Cores: Alternative Approaches to Stone Tool Analysis. Newcastle: Cambridge Scholars Publishing, pp. 267-276.

Moncel, M.-H. (2003a): "Tata (Hongrie). Un assemblage microlithique du début du Pléistocène supérieur en Europe centrale", L'Anthropologie, 107, pp. 117-151.

http://dx.doi.org/10.1016/S0003-5521(02)00005-5

MONCEL, M.-H. (2003b): L'exploitation de l'espace et la mobilité des groupes humains au travers des assemblages lithiques à la fin du Pléistocène moyen et au début du Pléistocène supérieur. La moyenne vallée du
Rhône, entre Drôme et Ardèche, France. BAR Int. Ser., 1184. Oxford: Archaeopress.

Orians, G. H. y Pearson, N. E. (1979): "On the Theory of Central Place Foraging”. En HoRn, D. J.; Stairs, G. R. y Mitchell, R. D. (eds.): Analysis of Ecological Systems. Columbia: Ohio State University Press, pp. 155-177.

Pelegrin, J.; Karlin, C. y Bodu, P. (1988): "Chaînes opératoires: un outil pour le préhistorien”. En TIXIER, J. (ed.): Technologie Prehistorique. Paris: CNRS, pp. 1-153.

Querol, M. Á. (1985): "Los sistemas de aprovechamiento: un modelo para la interpretación y el estudio de los grupos prehistóricos", Arqueologia, 12, pp. 20-33.

Salvayre, S. (1983): Géologie des Pyrénées Orientales, essai de synthèse. Perpignan: Sofreix.

TURQ, A. (2000): "Paléolithique inferieur et moyen entre Dordogne et Lot", Paléo, supplément, 2, pp. 1-456. 